\title{
The MJO in a Coarse-Resolution GCM with a Stochastic Multicloud Parameterization
}

\author{
QIANG DENG \\ Center for Prototype Climate Modeling, New York University Abu Dhabi, Abu Dhabi, United Arab Emirates \\ BOUALEM KHOUIDER \\ Department of Mathematics and Statistics, University of Victoria, Victoria, British Columbia, Canada \\ ANDREW J. MAJDA \\ Department of Mathematics, and Center for Atmosphere and Ocean Sciences, Courant Institute for \\ Mathematical Sciences, New York University, New York, New York, and Center for Prototype \\ Climate Modeling, New York University Abu Dhabi, Abu Dhabi, United Arab Emirates
}

(Manuscript received 23 April 2014, in final form 29 August 2014)

\begin{abstract}
The representation of the Madden-Julian oscillation (MJO) is still a challenge for numerical weather prediction and general circulation models (GCMs) because of the inadequate treatment of convection and the associated interactions across scales by the underlying cumulus parameterizations. One new promising direction is the use of the stochastic multicloud model (SMCM) that has been designed specifically to capture the missing variability due to unresolved processes of convection and their impact on the large-scale flow. The SMCM specifically models the area fractions of the three cloud types (congestus, deep, and stratiform) that characterize organized convective systems on all scales. The SMCM captures the stochastic behavior of these three cloud types via a judiciously constructed Markov birth-death process using a particle interacting lattice model. The SMCM has been successfully applied for convectively coupled waves in a simplified primitive equation model and validated against radar data of tropical precipitation. In this work, the authors use for the first time the SMCM in a GCM. The authors build on previous work of coupling the High-Order Methods Modeling Environment (HOMME) NCAR GCM to a simple multicloud model. The authors tested the new SMCM-HOMME model in the parameter regime considered previously and found that the stochastic model drastically improves the results of the deterministic model. Clear MJO-like structures with many realistic features from nature are reproduced by SMCM-HOMME in the physically relevant parameter regime including wave trains of MJOs that organize intermittently in time. Also one of the caveats of the deterministic simulation of requiring a doubling of the moisture background is not required anymore.
\end{abstract}

\section{Introduction}

Global climate models (GCMs) are large computer codes based on a discretization of some sort of the governing primitive equations of atmosphere-ocean fluid dynamics that are used routinely for operational climate and long range weather predictions and for research purposes. Owing to computing resources limitation, grid resolutions on the order of $100-200 \mathrm{~km}$ are

Corresponding author address: Dr. Boualem Khouider, Mathematics and Statistics, University of Victoria, P.O. Box 3045, STN CSC, Victoria BC V8W 3P4, Canada.

E-mail: khouider@math.uvic.ca typically used. On such coarse grids only synoptic$(\approx \geq 2000 \mathrm{~km})$ and planetary-scale features are represented (Krishnamurti et al. 2006). The effect of unresolved scales on the large-scale flow is accounted for through various recipes called parameterization (Stensrud 2007). The design and construction of reliable parameterization schemes for various processes such as land and sea surface atmospheric interactions, boundary layer turbulence, moist convection, cloud microphysics, radiation, orography, gravity waves, ocean mixing, etc. is an active area of research (Stensrud 2007). These parameterization schemes are based on various theories and on our basic understanding of the underlying processes. The parameterization of moist convection, also known as

DOI: $10.1175 / J A S-D-14-0120.1$

(C) 2015 American Meteorological Society 
cumulus parameterization, is one of the most challenging problems in climate modeling research owing to a poor understanding of the interactions across scales of organized convective systems (Moncrieff and Klinker 1997; Lin et al. 2006; Majda 2007) ranging from the convective cell of $1-10 \mathrm{~km}$, to mesoscale systems of $50-500 \mathrm{~km}$, to synopticand planetary-scale systems such as convectively coupled equatorial waves (CCWs) and the Madden-Julian oscillation (MJO) (Madden and Julian 1972; Wheeler and Kiladis 1999; Khouider et al. 2013).

To capture the missing variability in GCMs due to unresolved processes, particularly organized tropical convection, many strategies for incorporating random variables into the otherwise deterministic parameterization schemes have been proposed. For instance, Buizza et al. (1999) applied uniformly distributed multiplicative coefficients into the parameterized tendencies while Lin and Neelin (2003) included a random perturbation to the convective available potential energy (CAPE) tendency in a mass flux parameterization. Moreover, Plant and Craig (2008) used a Boltzmanntype equilibrium distribution for the ensemble cloud base mass flux combined with a Poisson processes for the number of plumes in the ensemble. Following earlier work (Majda and Khouider 2002; Khouider et al. 2003), Khouider et al. (2010, hereafter KBM10) used the nonequilibrium statistical mechanics framework of interacting particle lattice systems to propose a stochastic multicloud model (SMCM) for organized tropical convection. The SMCM is slightly modified and used in Frenkel et al. (2012, hereafter FMK12, 2013, hereafter FMK13) for simulations of convectively coupled gravity waves and organized convection in a simplified setting of 2D flows over the equator. In the SMCM, a rectangular lattice overlays each GCM grid box and each lattice site is assumed to be either occupied by a cloud of a certain type (congestus, deep, or stratiform) or it is a clear sky site. The so-defined convective elements interact with the large-scale environment and with each other through CAPE and middle troposphere dryness. In MJO as well as synoptic and mesoscale organized convective events, the life cycle of the cloud morphology is characterized by transitions from congestus to deep convection then to stratiform (Johnson et al. 1999; Mapes et al. 2006; Del Genio et al. 2012). In the SMCM, the constraint of this one-way route from congestus to deep to stratiform is implemented through a judicious choice of the probability transition rates. For the sake of simplicity (KBM10; FMK12; FMK13), local interactions between the lattice sites are ignored so that a multidimensional birth-death process is derived for the cloud area fractions, of the three cloud types, that can be easily and efficiently evolved in time and coupled to an existing deterministic cumulus parameterization. Nonetheless, a systematic coarsegraining approximation strategy (Katsoulakis et al. 2003; Khouider et al. 2003) leading to a similar birthdeath process is presented and used in Khouider (2014) to incorporate local interactions in the SMCM.

The transition rates depend on some large-scale measures of convective instability (CAPE) and middle troposphere moistness through Arrhenius-like activationenergy functions depending on a set of transition time scale parameters. In KBM10, FMK12, and FMK13, ad hoc values of these transition time scales, based solely on physical intuition, were successfully used to reproduce qualitatively the stochastic behavior of tropical convection and convectively coupled waves. On the other hand, in Peters et al. (2013), the SMCM is shown to reproduce some important features of the precipitation statistics of times series of radar data from two tropical stations. In this first attempt of deducing the SMCM parameters from observations, the transition time scales were constrained so that the large-scale distribution of the mean cloud area fractions matchs qualitatively the radar data. However, they did not perform well in our GCM simulations. This may be due to limitations in the radar data, namely regarding the ability to capture cumulus congestus clouds. Also note that Peters et al. (2013) have used vertical motion as a predictor instead of CAPE, unlike the present study. Another possibility for this poor performance of the Peters et al. (2013) parameters may be due to our aquaplanet setting given that considerable land-sea interactions are exhibited at the two observation sites. Nonetheless, a more rigorous-Bayesian inference method for inferring these parameters from data is developed and validated, against synthetic data, in De La Chevrotiere et al. (2014) but it is yet to be applied for real data. We thus rely on the strategy adopted in FMK13 to select our benchmark time scale parameters in an ad hoc fashion.

The continued theoretical and modeling efforts in MJO research of the last few decades were not fruitless. Remarkable improvements in both the understanding and the simulation of MJO in GCMs have been achieved (Zhang 2005; Majda and Stechmann 2009; Subramanian et al. 2011; Zhang et al. 2013a; Del Genio et al. 2012; Crueger et al. 2013). However, there are still some challenges in terms of reproducing some of the key observed features of MJO, including its chaotic initiation, propagation, and demise. Most GCM MJOs are very sensitive to initial and boundary (surface coupling) conditions (Lin et al. 2006; Liu et al. 2009; Kim et al. 2009). While it is still debated whether the MJO is a distinguishable mode of variability of the tropical atmosphere, there is a consensus that low- to midlevel moisture play a key role in the dynamics and in the GCM simulations of the MJO (Kikuchi and Takayabu 2004; Del Genio et al. 2012). 
As opposed to traditional plume-based and large-scale forcing-type parameterization, a new look into the cumulus parameterization problem was provided through the multicloud model of Khouider and Majda (2006a,b). Three cloud types - congestus, deep, and stratiform - are observed to characterize organized tropical convective systems (Lin and Johnson 1996; Johnson et al. 1999; Lin et al. 2004), and the multicloud model is designed based on a judicious representation of the dynamics associate with these cloud types, following previous work (Mapes 2000; Majda and Shefter 2001; Majda et al. 2004). This deterministic version of the multicloud parameterization (DMCM) has been successfully coupled to the nextgeneration National Center for Atmospheric Research (NCAR) GCM, the High-Order Methods Modeling Environment (HOMME) (Khouider et al. 2011, hereafter KSMT11), and used to investigate the initiation and dynamics of MJO in a warm pool background (Ajayamohan et al. 2013). HOMME is a spectral element model based on a cubed sphere discretization in the horizontal and finite differences in $\sigma$ coordinate in the vertical. It is, particularly, a highly scalable model in terms of parallelism and has many desired features in terms of simulating kinetic energy spectra of dry-atmospheric dynamics. More details can be found in KSMT11 and references therein.

Here, for the first time the SMCM is coupled to a GCM. We build on previous work of KSMT11, where the HOMME NCAR GCM is coupled to the DMCM. The same parameter regime of KSMT11 is tested. As in FMK12, we found that the stochastic model drastically improves the results of the DMCM-HOMME model. Clear MJO-like structures with many realistic features from nature are produced. Also one of the caveats of the deterministic simulation of requiring a doubling of the moisture background is not required anymore. The SMCM is coupled to the existing deterministic multicloud parameterization (KSMT11) through the stochastically evolving cloud area fractions, which are used in the heating closures to allow consistent transitions between cloud types. As illustrated in Fig. 1, a similar coupling can be achieved with essentially any existing cumulus parameterization by modifying the mass flux and/or the detrainment levels for plume-based parameterization, for example.

The paper is organized as follows. In section 2, we briefly review the implementation of the multicloud model in the HOMME GCM and the most salient features of the stochastic multicloud parameterization. Moreover, a benchmark simulation obtained by the deterministic multicloud-HOMME is shown to highlight the drastic improvement of the stochastic parameterization. Section 3 is devoted to a simulation using the SMCM-HOMME in the parameter regime of the benchmark deterministic simulation of section 2, where
Stochastic Multi-cloud Model to inform cumulus parameterization:

represent the missing sub-grid scale variability

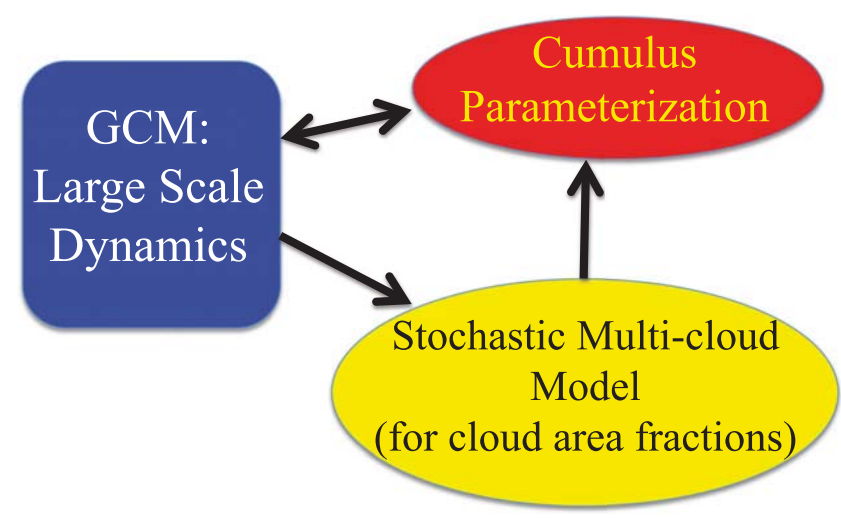

FIG. 1. The schematic of the implementation of the stochastic multicloud parameterization in GCM.

the results are presented and analyzed. Detailed analysis confirms that the main features of MJOs have been captured by the stochastic parameterization. Sensitivity tests to key parameters of the SMCM such as the probability transition time scales and the number of lattice sites together with a statistical analysis are presented in section 4. Finally, a concluding summary and a discussion are provided in section 5 .

\section{Model setup and deterministic benchmark simulation}

In this section we briefly review the implementation of the multicloud model in the HOMME GCM (KSMT11) together with some of the most salient features of the stochastic multicloud parameterization (KBM10; FMK12; FMK13). A more complete discussion of the stochastic model and multicloud framework can be found in the original papers. To highlight the drastic improvement using the stochastic parameterization, a benchmark deterministic multicloud-HOMME simulation is also presented in this section.

\section{a. The multicloud model in HOMME GCM}

As mentioned above, the DMCM parameterization is coupled to the HOMME dynamical core in KSMT11 and used to simulate convectively coupled equatorial waves and the MJO [see also Ajayamohan et al. (2013)]. To ease the understanding of the coupling of the SMCM (to be discussed in more detail below) to the HOMME-MCM model, we first present a brief outline on how the DMCM is implemented in HOMME. Further details can be found in KSMT11.

The HOMME dynamical core is considered in an aquaplanet setting where all physical processes were 
TABLE 1. Convective heating closures for the stochastic and deterministic parameterization.

\begin{tabular}{ll}
\hline \hline \multicolumn{1}{c}{ Stochastic } & \multicolumn{1}{c}{ Deterministic } \\
\hline$H_{c}=\sigma_{c} \frac{\alpha_{c} \bar{\alpha}}{H_{m}} \sqrt{\mathrm{CAPE}_{l}^{+}}$ & $\frac{\partial H_{c}}{\partial t}=\frac{1}{\tau_{c}}\left(\Lambda \alpha_{c} Q_{c}^{+}-H_{c}\right)$ \\
$H_{d}=\left\{\sigma_{d} \bar{Q}+\frac{1}{\tau_{c}\left(\sigma_{d}\right)}\left[a_{1} \theta_{\mathrm{eb}}+a_{2} q-a_{0}\left(\theta_{1}+\gamma_{2} \theta_{2}\right)\right]\right\}^{+}$ & $H_{d}=(1-\Lambda) Q_{d}^{+}$ \\
$H_{s}=\alpha_{s}\left\{\sigma_{s} \bar{Q}+\frac{1}{\tau_{c}\left(\sigma_{s}\right)}\left[a_{1} \theta_{\mathrm{eb}}+a_{2} q-a_{0}\left(\theta_{1}+\gamma_{2} \theta_{2}\right)\right]\right\}^{+}$ & $\frac{\partial H_{s}}{\partial t}=\frac{1}{\tau_{s}}\left(\alpha_{s} H_{d}-H_{s}\right)$ \\
$\tau_{c}\left(\sigma_{d}\right)=\frac{\bar{\sigma}_{d}}{\sigma_{d}} \tau_{\mathrm{conv}}$ & $Q_{c}=\bar{Q}+\frac{1}{\tau_{\mathrm{conv}}}\left[\theta_{\mathrm{eb}}-a_{0}^{\prime}\left(\theta_{1}+\gamma_{2}^{\prime} \theta_{2}\right)\right]$ \\
$\tau_{c}\left(\sigma_{s}\right)=\frac{\bar{\sigma}_{s}}{\sigma_{s}} \tau_{\mathrm{conv}}$ & $Q_{d}=\bar{Q}+\frac{1}{\tau_{\mathrm{conv}}}\left[a_{1} \theta_{\mathrm{eb}}+a_{2} q-a_{0}\left(\theta_{1}+\gamma_{2} \theta_{2}\right)\right]$ \\
& $\Lambda=\left\{\begin{array}{lll}0, & \text { if } & \theta_{\mathrm{eb}}-\theta_{\mathrm{em}} \leq \theta^{-} \\
1, & \text { if } & \theta_{\mathrm{eb}}-\theta_{\mathrm{em}} \geq \theta^{+} \\
\text {Linear continuous, } & \text { otherwise }\end{array}\right.$ \\
\hline
\end{tabular}

turned off except for upper-tropospheric damping of gravity waves and rigid surface boundary conditions. The MCM alone drives the otherwise dry dynamics through the parameterized latent heating (KSMT11):

$$
Q_{c}=H_{d} \tilde{\psi}_{1}(p)+\left(H_{c}-H_{s}\right) \tilde{\psi}_{2}(p),
$$

where $H_{d}, H_{c}, H_{s}$ are the heating rates associated with deep, congestus, and stratiform heating, respectively, while $\tilde{\psi}_{1}, \tilde{\psi}_{2}$ are the potential temperature vertical profiles associated with the first and second baroclinic modes (Kasahara and Puri 1981). The basis functions were carefully normalized and truncated to zero above $200 \mathrm{hPa}$ to limit the latent heating in the troposphere (KSMT11). They are based on a reference basic state obtained from the Global Atmospheric Research Program (GARP) Atlantic Tropical Experiment (GATE) sounding profile (Grabowski 2002). While their specific shape is not reproduced here, they roughly resemble a half sine and full sine, respectively, so that deep convection heats the whole atmospheric column and peaks in the middle of the troposphere while congestus (stratiform) clouds heat the lower (upper) troposphere and cool the upper (lower) troposphere. More details about the physical justifications for such heating profiles are given in Khouider and Majda (2006a,b) and the many other subsequent papers on the MCM.

To ensure radiative convective equilibrium (RCE) with respect to the tropical sounding, which is used as initial conditions, we impose a radiative cooling with the same vertical profile as the cumulus heating:

$$
Q_{R}=Q_{R, 1}^{0} \tilde{\psi}_{1}(p)+Q_{R, 2}^{0} \tilde{\psi}_{2}(p),
$$

where $Q_{R, 1}^{0}$ and $Q_{R, 2}^{0}$ are the constant radiative cooling strength associated with the first and second baroclinic modes, respectively.
It is also worthwhile noting that, in order to prevent any interactions with extratropical waves, a mask is superimposed on the temperature tendency equation to limit the parameterized latent heating and imposed cooling rates to an equatorial belt between $30^{\circ} \mathrm{S}$ and $30^{\circ} \mathrm{N}$. The whole system is relaxed toward the tropical sounding on a 50-day time scale to maintain RCE outside the tropical belt.

The coupled system is augmented with two 2D scalar equations for the boundary layer equivalent potential temperature $\theta_{\mathrm{eb}}$ and vertical average moisture $q$, which are used to derive closures for the heating rates $H_{d}, H_{c}$, and $H_{s}$. They are given by

$$
\frac{\partial \theta_{\mathrm{eb}}}{\partial t}+\mathbf{u}\left(x, y, p_{1}, t\right) \cdot \nabla \theta_{\mathrm{eb}}=\frac{1}{h} E_{s}-\frac{1}{h} D \quad \text { and }
$$

TABLE 2. Transition rates and time scales in the stochastic parameterization.

\begin{tabular}{llr}
\hline \multicolumn{1}{c}{ Transition } & \multicolumn{1}{c}{$\begin{array}{c}\text { Transition } \\
\text { rate }\end{array}$} & Time scale (h) \\
\hline $\begin{array}{l}\text { Formation of } \\
\text { congestus }\end{array}$ & $R_{01}=\frac{1}{\tau_{01}} \Gamma\left(C_{l}\right) \Gamma(D)$ & $\tau_{01}=1 \tau_{\text {grid }}$ \\
Decay of congestus & $R_{10}=\frac{1}{\tau_{10}} \Gamma(D)$ & $\tau_{10}=1 \tau_{\text {grid }}$ \\
$\begin{array}{lll}\text { Conversion of } \\
\text { congestus to deep }\end{array}$ & $R_{12}=\frac{1}{\tau_{12}} \Gamma(C)[1-\Gamma(D)]$ & $\tau_{12}=1 \tau_{\text {grid }}$ \\
$\begin{array}{ll}\text { Formation of deep } \\
\text { Conversion of deep } \\
\text { to stratiform }\end{array}$ & $R_{02}=\frac{1}{\tau_{02}} \Gamma(C)[1-\Gamma(D)]$ & $\tau_{02}=3 \tau_{\text {grid }}$ \\
Decay of deep & $R_{20}=\frac{1}{\tau_{20}}[1-\Gamma(C)]$ & $\tau_{23}=3 \tau_{\text {grid }}$ \\
& & \\
Decay of stratiform & $R_{30}=\frac{1}{\tau_{30}}$ & $\tau_{20}=3 \tau_{\text {grid }}$
\end{tabular}


TABLE 3. List of multicloud parameters for the deterministic and stochastic simulations. The values in parentheses for $\tilde{Q}_{j}$ correspond to a normalization with the $L_{2}$ norm of the basis function $\phi_{j}$ that enters in the projections of the horizontal velocity field.

\begin{tabular}{|c|c|c|}
\hline Parameter & Value & Description \\
\hline $\begin{array}{l}\text { No. of elements for } \\
\text { HOMME GCM }\end{array}$ & 20 & \\
\hline Time step & $30 \mathrm{~s}$ & \\
\hline Vertical levels & 26 & \\
\hline Tropical mask & $\begin{array}{l}\text { Smoothed Heaviside function } \\
\text { with slope } k=10 \text { and } \\
y_{0}= \pm 30^{\circ}\end{array}$ & \\
\hline Surface forcing & Uniform SST & \\
\hline $\begin{array}{l}\text { Temperature } \\
\text { background }\end{array}$ & GATE & \\
\hline $\begin{array}{l}\text { Background moisture } \\
\text { gradient profile }\end{array}$ & GATE & \\
\hline$\tilde{Q}_{1}$ & $38.47(6.15) \mathrm{K}$ & First baroclinic projection of the background moisture gradient in Eq. (4) \\
\hline$\tilde{Q}_{2}$ & $38.35(3.1) \mathrm{K}$ & $\begin{array}{l}\text { Second baroclinic projection of the background moisture gradient in } \\
\text { Eq. (4) }\end{array}$ \\
\hline$Q_{R, 1}^{0}$ & $1 \mathrm{~K}_{\text {day }}{ }^{-1}$ & First baroclinic radiative cooling rate in Eq. (2) \\
\hline $\bar{\theta}_{\mathrm{eb}}-\bar{\theta}_{\mathrm{em}}$ & $11.00 \mathrm{~K}$ & Discrepancy between $\theta_{\mathrm{eb}}$ and $\theta_{\mathrm{em}}$ at RCE in Eq. (6) \\
\hline$\theta_{\mathrm{eb}}^{*}-\bar{\theta}_{\mathrm{eb}}$ & $10.00 \mathrm{~K}$ & Discrepancy between saturation and actual $\theta_{\mathrm{eb}}$ at RCE in Eq. (6) \\
\hline$a_{1} / a_{2}$ & $0.1 / 0.9$ & Relative contribution of $\theta_{\mathrm{eb}}-q$ to deep convection in Table 1 \\
\hline$a_{0}$ & 0.5 & $\begin{array}{l}\text { Dry convective buoyancy frequency in deep and congestus heating } \\
\text { in Table } 1\end{array}$ \\
\hline$\gamma_{2} / \gamma_{2}^{\prime}$ & $0.25 / 0.6$ & 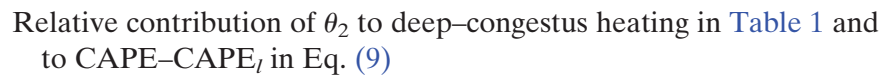 \\
\hline$\mu$ & 0.2 & Relative contribution of stratiform and congestus to downdrafts in Eq. (6) \\
\hline$\alpha_{c} / \alpha_{s}$ & $0.25 / 0.5$ & Congestus-stratiform adjustment coefficient in Table 1 \\
\hline$\tau_{c} / \tau_{s}$ & $1 \mathrm{~h} / 3 \mathrm{~h}$ & Congestus-stratiform adjustment time scale in Table 1 \\
\hline$\tau_{\text {conv }}$ & $2 \mathrm{~h}$ & Convective time scale in Table 1 \\
\hline$R$ & $320 \mathrm{~J} \mathrm{~kg}^{-1} \mathrm{~K}^{-1}$ & CAPE constant in Eq. (9) \\
\hline$\gamma$ & 1.7 & Contribution of $\theta_{1}$ to CAPE anomalies in Eq. (9) \\
\hline
\end{tabular}

$$
\begin{aligned}
\frac{\partial q}{\partial t} & +\nabla \cdot\left[q\left(\overline{\mathbf{u}}+\mathbf{u}_{1}+\tilde{\alpha} \mathbf{u}_{2}\right)\right]+\tilde{Q}_{1} \nabla \cdot \mathbf{u}_{1}+\tilde{Q}_{2} \nabla \cdot \mathbf{u}_{2} \\
& =-P+\langle E\rangle,
\end{aligned}
$$

where

$$
\tilde{Q}_{j}=\int_{p_{T}}^{p_{B}} \frac{d \tilde{Q}(p)}{d p} \psi_{j}(p) d p, \quad j=1,2
$$

are two "parameters" representing the background moisture profile $\tilde{Q}$ associated with the reference tropical sounding. Here, $p_{1}$ is the pressure at the lowest grid level above the surface and $h=500 \mathrm{~m}$ is the height of the atmospheric boundary layer, which is assumed to be constant. The quantity $E_{s}$ is the evaporation from the sea surface and $D$ is the downdraft mass flux:

$$
\begin{aligned}
\frac{1}{h} E_{s} & =\frac{1}{\tau_{e}}\left(\theta_{\mathrm{eb}}^{*}-\theta_{\mathrm{eb}}\right), \\
D & =\frac{m_{0}}{Q_{R, 1}^{0}}\left[Q_{R, 1}^{0}+\mu\left(H_{s}-H_{c}\right)\right]^{+}\left(\theta_{\mathrm{eb}}-\theta_{\mathrm{em}}\right),
\end{aligned}
$$

where $\theta_{\mathrm{eb}}^{*}$ is the boundary layer saturation equivalent potential temperature and $\theta_{\mathrm{em}}=q+\left\langle\psi_{1}\right\rangle\left(\theta_{1}+\alpha_{2} \theta_{2}\right)$ is the middle-tropospheric equivalent potential temperature. The angle brackets $\langle\cdot\rangle$ indicate the vertical average over the free troposphere.

The surface precipitation and evaporation rates are set as bellow to guarantee conservation of vertically integrated moist static energy in the system (Khouider and Majda 2006a; KSMT11):

$$
P=\frac{1}{p_{B}-p_{T}} \int_{p_{T}}^{p_{B}} Q_{c}(x, y, p, t) d p, \quad\langle E\rangle=\frac{D}{H} .
$$

Note that the heating basis functions are fixed during the simulation and the heating rates will be updated through the closure equations in Table 1 . The resolved large-scale variables are used to calculate the total available "bulk energy" for each cloud type, and a moisture switch function $\Lambda$ is introduced to modulate the strength of the deep convection and allow smooth transitions between congestus and deep convection. The $\Lambda$ only depends on the moistness and 

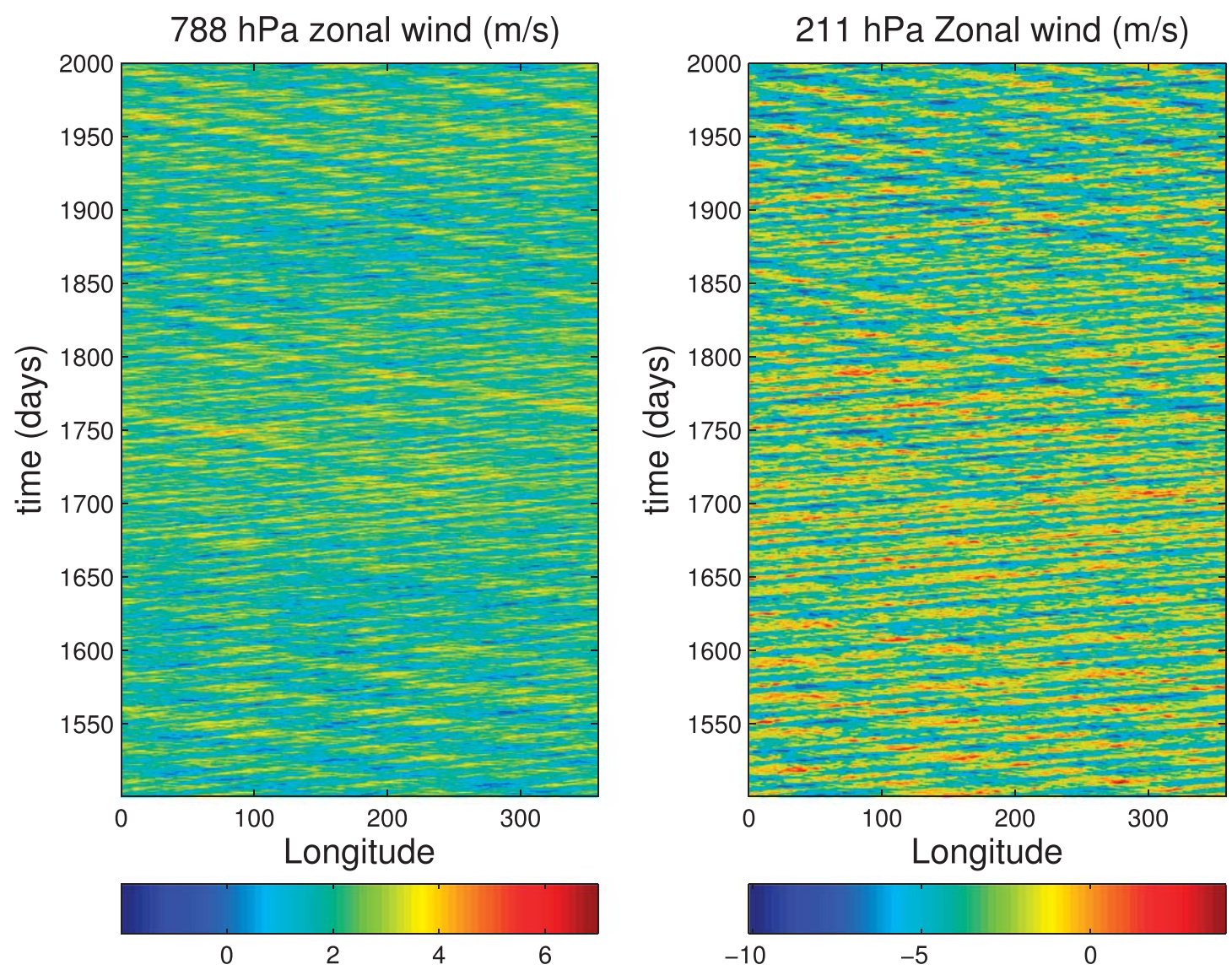

FIG. 2. Hovmöller diagrams of (left) 788-and (right) 211-hPa zonal wind for the last 500 days of the benchmark deterministic multicloud-HOMME simulation with $\alpha_{s}=0.5$ and non-doubled GATE moisture background constant $\tilde{Q}_{1}$ and $\tilde{Q}_{2}$ as shown in Table 3. Used for the comparison with the SMCM-HOMME simulations.

dryness of the middle troposphere and is totally deterministic.

\section{b. The stochastic multicloud model}

As mentioned earlier, the SMCM is introduced in KBM10 and used successfully in FMK12 and FMK13 to study organized convection and convectively coupled gravity waves for 2D flows over the equator. While the details of this model are found in these papers, here we briefly illustrate some of its main features for the sake of completeness. In essence, the SMCM is a (stochastic) model for the area fractions associated with the three cloud types, congestus, deep, and stratiform.

A rectangular lattice of $n \times n$ lattice sites is overlaid over each GCM horizontal grid box. Each lattice site is assumed to be either clear sky or occupied by a congestus, deep, or a stratiform cloud. The fraction of sites occupied by a given cloud type is thus regarded as the corresponding cloud area fraction within the given GCM grid box. To evolve the cloud area fractions in time, the individual cloud sites make random transitions from one state to another according to intuitive probability rules depending on the large-scale GCM variables following observations (Johnson et al. 1999; Mapes 2000). The transition probabilities are given as functions of the convective available potential energy integrated over the whole troposphere (CAPE), the convectively available energy integrated over the lower troposphere $\left(\mathrm{CAPE}_{l}\right)$, and the dryness of the midtroposphere, according to the following intuitive rules (KBM10).

(i) If low-level CAPE is positive and the middle troposphere is dry, then a clear site turns into a congestus site with high probability.

(ii) If CAPE is positive and the middle troposphere is moist, then a congestus or clear sky site turns into a deep convective site with high probability.

(iii) A deep convective site turns into a stratiform site with (a fixed) high probability.

(iv) All three cloud types decay naturally to clear sky at some fixed rates.

(v) All other transitions are assumed to have negligible probabilities. 

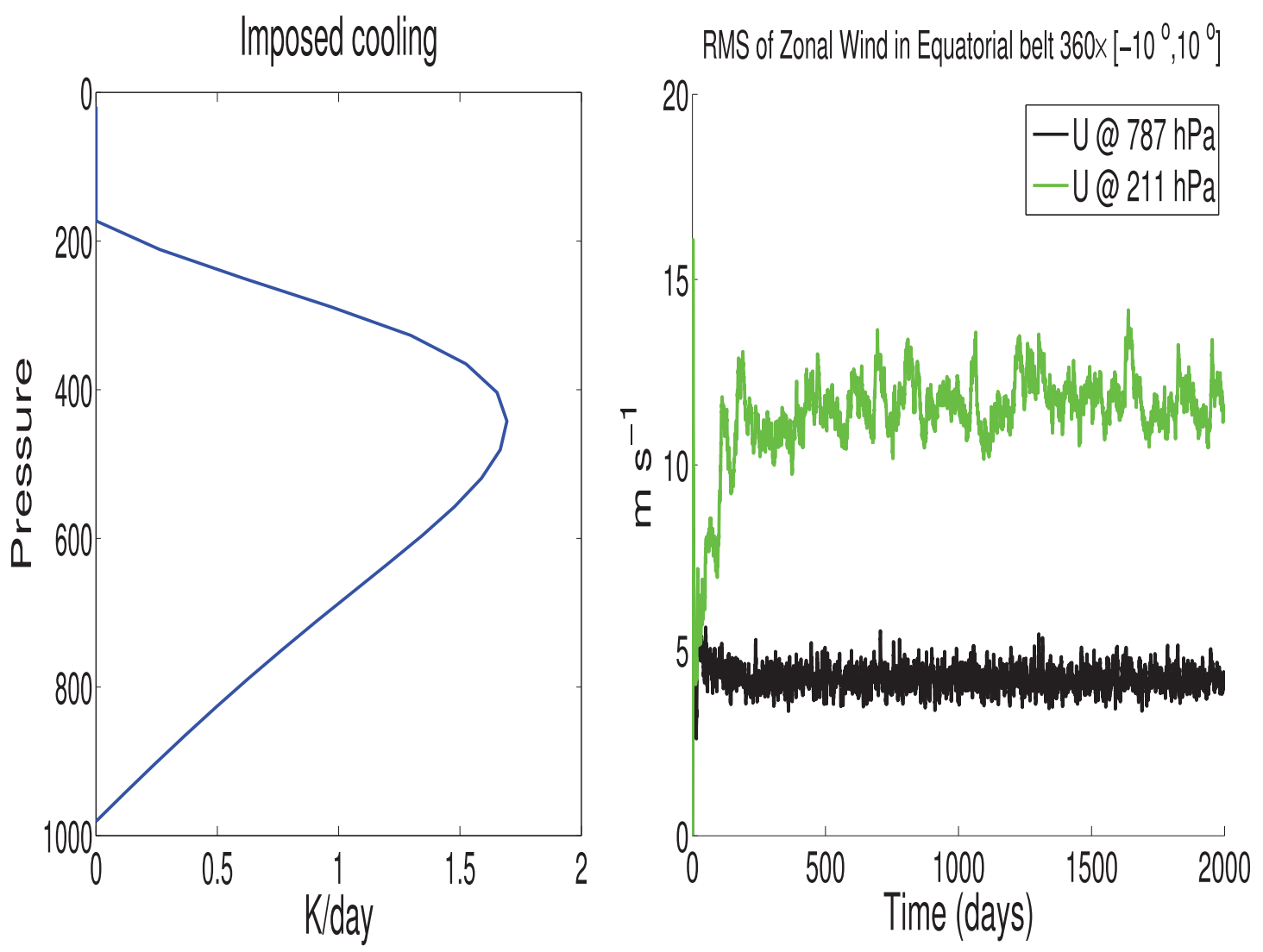

FIG. 3. (left) Vertical profile of the imposed radiative cooling and (right) time series of the horizontal rms of the zonal velocity in the lower and upper troposphere, averaged over the equatorial belt $10^{\circ} \mathrm{S}-10^{\circ} \mathrm{N}$, for the SMCMHOMME simulation with $n=20$ and $\tau_{\text {grid }}=4$.

The corresponding transition rates are formulated in Table 2 in terms of the Arrhenius-type function:

$$
\Gamma(x)= \begin{cases}1-\exp (-x), & \text { if } x>0 \\ 0, & \text { otherwise }\end{cases}
$$

The remaining parameters are listed in Tables 2 and 3 and a more detailed discussion can be found in the original papers KBM10, FMK12, and FMK13. In Table 2, $C_{1}, C$, and $D$ denote rescaled values of low-level CAPE, CAPE, and dryness:

$$
\begin{aligned}
C_{l} & =\mathrm{CAPE}_{l} / \mathrm{CAPE}_{0}, \quad C=\mathrm{CAPE} / \mathrm{CAPE}_{0}, \quad D=\left(\theta_{\mathrm{eb}}-\theta_{\mathrm{em}}\right) / T_{0}, \\
\mathrm{CAPE} & =\overline{\mathrm{CAPE}}+R\left[\theta_{\mathrm{eb}}-\gamma\left(\theta_{1}+\gamma_{2} \theta_{2}\right)\right], \quad \mathrm{CAPE}_{l}=\overline{\mathrm{CAPE}}+R\left[\theta_{\mathrm{eb}}-\gamma\left(\theta_{1}+\gamma_{2}^{\prime} \theta_{2}\right)\right] .
\end{aligned}
$$

The reference values $\mathrm{CAPE}_{0}=400 \mathrm{~J} \mathrm{~kg}^{-1}$ and $T_{0}=$ $30 \mathrm{~K}$ are used. Moreover, $\tau_{j k}$, with $j$ and $k=0,1,2,3$ are a set of parameters that set the time scales of the individual transitions. As already mentioned, these parameters would ideally be obtained from observations (De La Chevrotiere et al. 2014), but because of the lack of such values at this moment, we rely on arbitrary values motivated solely by intuition as done in FMK12 and FMK13. Here, we adapt the strategy of FMK13 of making such intuitive values depending on a single parameter $\tau_{\text {grid }}$ to take into account the ratio between the GCM grid resolution and the size of the underlying lattice.
In the present case, where the transition rates are assumed to depend only on the resolved large-scale variables (i.e., local interactions between individual lattice sites are ignored), the stochastic dynamics of the area fractions (or the coverage numbers) are known exactly, in closed form, without any further approximation (KBM10). Such coarse-graining procedure is not at all trivial when the local interactions are included. Nonetheless, an approximate solution is provided in Khouider (2014) for the case of nearest neighbor interactions, which interestingly is sufficient to allow interactions between nearest GCM grid boxes. Such column interactions 

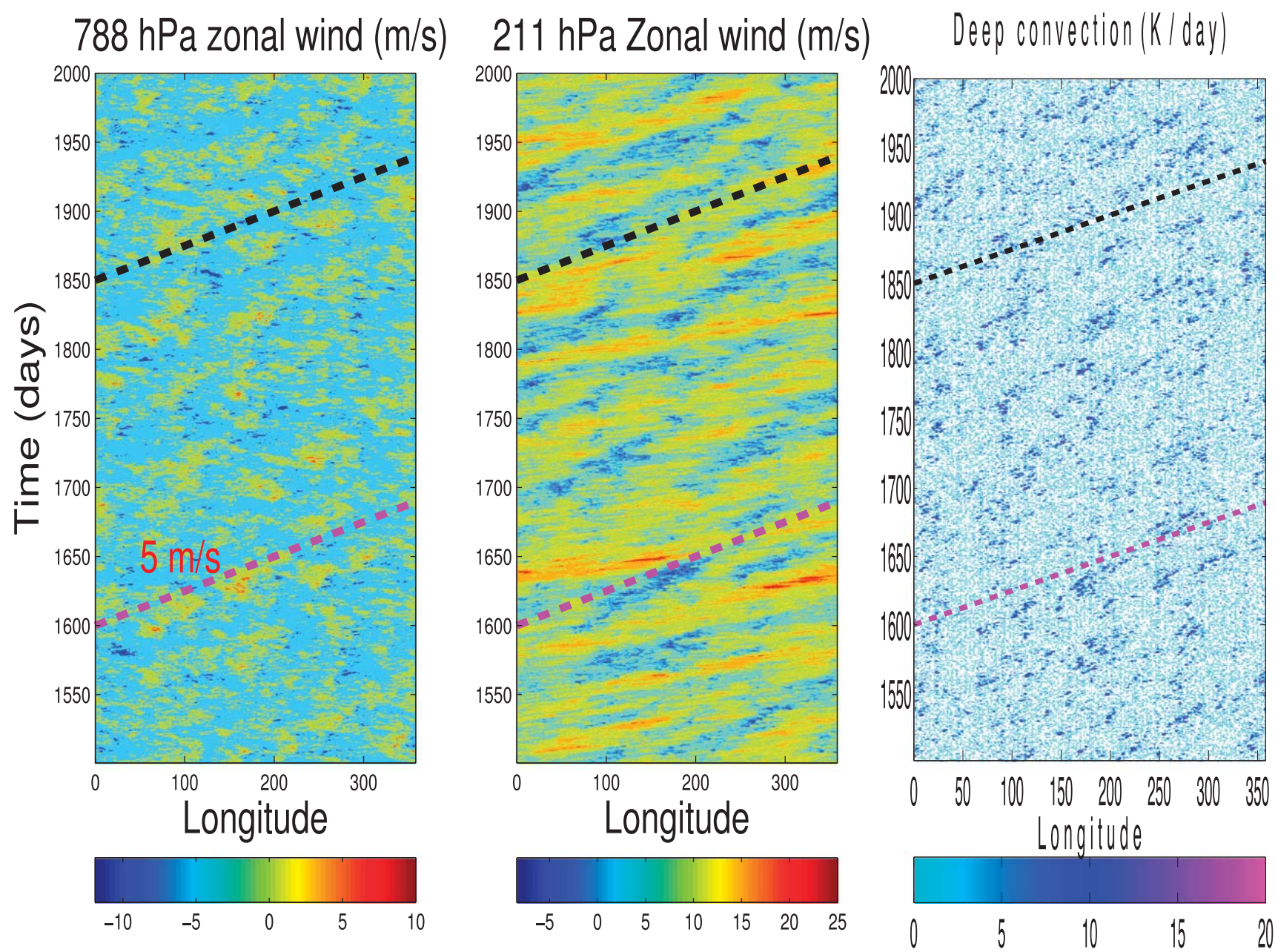

FIG. 4. Hovmöller diagrams of (left) 788- and (middle) 211-hPa zonal wind, and (right) deep convection $H_{d}$ averaged between $10^{\circ} \mathrm{S}-10^{\circ} \mathrm{N}$ for the last 500 days of the SMCM-HOMME simulation with $n=20$ and $\tau_{\text {grid }}=4$. A single (wavenumber 1 ) MJO-like event is marked by the magenta dashed lines starting at 1600 days and a train of two (wavenumber 2) MJO-like events is marked by the black dashed lines. All move eastward at roughly $5 \mathrm{~m} \mathrm{~s}^{-1}$.

of the cumulus parameterization may be important for seamless, high-resolution climate or weather predictionsimulations done in the so-called gray zone between 20 and $40 \mathrm{~km}$.

In summary, the coarse-graining procedure yields a multidimensional birth-death process for the corresponding three cloud-type area fractions (Katsoulakis et al. 2003; Khouider et al. 2003) that is evolved in time using Gillespie's exact algorithm (Gillespie 1975, 1977) without any significant computational overhead. Let $\Delta t$ be the time step of the GCM. To evolve the state of the SMCM (i.e., the cloud area fractions) from time $t$ to time $t+\Delta t$, at each GCM grid box, first, the first (random) transition time $\tau>0$ is sampled from the corresponding exponential distribution. If $\tau>\Delta t$, then the area fractions in the given GCM grid box remain unchanged. Otherwise, the most likely transition is found by choosing accordingly a second random number. The process is then repeated as necessary, with a new target time increment $\Delta t-\tau$, etc.

\section{c. A benchmark deterministic multicloud-HOMME simulation}

It is found in KSMT11 that in the MCM-HOMME model, the background vertical gradient of the moisture $\tilde{Q}(p)(5)$ and the stratiform fraction parameter $\alpha_{s}$ are two important control parameters that affect the type of waves that are produced by the aquaplanet simulations. One of the caveats of the DMCM-HOMME is the requirement of doubling the values of the moisture background parameters $\tilde{Q}_{j}$ associated with the GATE sounding to generate MJO-like waves (KSMT11). Three numerical simulations using different moisture gradients and different stratiform fractions were considered in KSMT11: two with the GATE $\tilde{Q}_{j}$ values and $\alpha_{s}=0.5$ and $\alpha_{s}=0.25$ and one with a doubling of the $\tilde{Q}_{j}$ 
values and $\alpha_{s}=0.25$. Only the case with a doubled moisture gradient resulted in a simulation with MJOlike disturbances. The simulation with the small stratiform fraction and a GATE moisture gradient resulted in an unrealistic planetary-scale (wavenumber-1) second baroclinic Kelvin wave and the one with a large stratiform fraction and a GATE moisture gradient gave rise to convectively coupled Rossby, Kelvin, and 2-day waves propagating through each other.

Here, we reproduce the DMCM-HOMME simulation with the weaker (GATE) background moisture gradient and a large stratiform fraction (Table 3 ) and use it as a benchmark for the stochastic simulation presented in the next section. As in KSMT11, the horizontal resolution is fixed to $20 \times 20$ spectral elements on each face of the cubed sphere yielding a resolution of about 240 grid points or a mesh size of $167 \mathrm{~km}$ along the equator. A time step of $30 \mathrm{~s}$ is used to resolve the convective processes.

We assume an aquaplanet with no land or topography (Grabowski 2002). The imposed cooling is determined by $Q_{R, 1}^{0}=1 \mathrm{~K}$ and the RCE solution corresponding to $\bar{\theta}_{\mathrm{eb}}-\bar{\theta}_{\mathrm{em}}=11 \mathrm{~K}$ (a moist RCE) and $\theta_{\mathrm{eb}}^{*}-\bar{\theta}_{\mathrm{eb}}=10 \mathrm{~K}$ (KSMT11). We assume an initial reference state of rest with a temperature profile inferred from the GATE sounding (Grabowski 2002; Grabowski and Moncrieff 2004) plus a small random perturbation. The numerical model is then evolved in time toward a statistical equilibrium for a period of 2000 days.

The Hovmöller diagrams of the zonal wind at top and bottom of the troposphere are shown in Fig. 2. While a detailed analyzed of this simulations are provided in KSMT11, we note here that it is characterized by very regular convectively coupled waves. No MJO events are produced and no intrinsic chaotic behavior as it occurs in nature is seen here. As shown in the next section, in the same parameter regime, the simulation with SMCMHOMME reproduces clear chaotic MJO-like events that change in character with high intermittency, as in nature.

\section{A typical stochastic simulation}

In this section, we present the simulation results of a typical stochastic simulation using the coupled SMCM-HOMME model. The simulation is performed under the same conditions as the benchmark simulation presented above except for the use of the stochastic closure equations in Table 1 instead of the deterministic ones. The number of lattice sites is set to $n \times n=400$ and $\tau_{\text {grid }}=4$. As pointed out in FMK13, $n$ and $\tau_{\text {grid }}$ are important parameters that affect the variability of the model; a large $\tau_{\text {grid }}$ or equivalently a small $n$ tends to

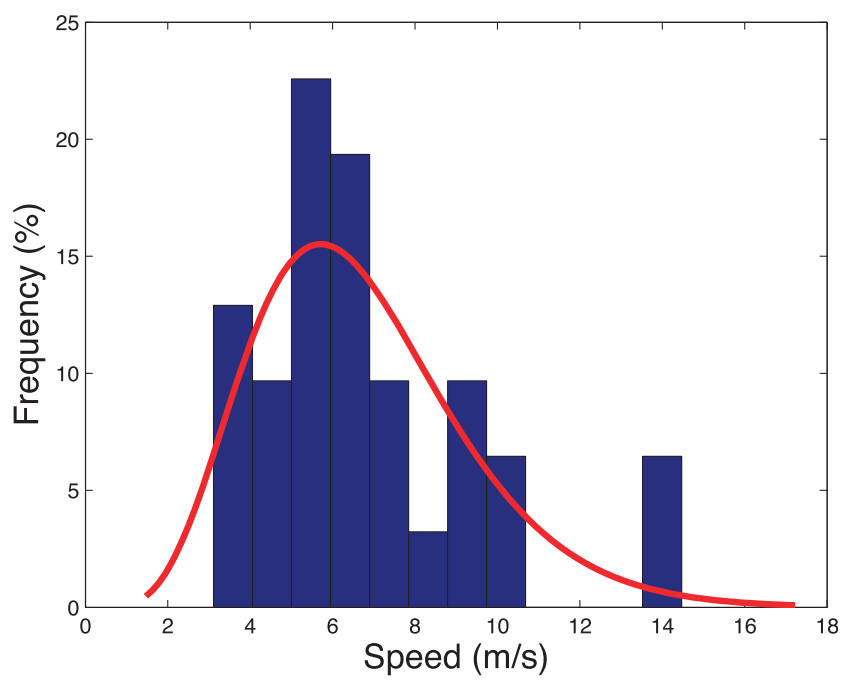

FIG. 5. The histogram (bars) and Gamma fit (curve) of the eastward propagation speeds of the $31 \mathrm{MJO}$-like events produced by the SMCM-HOMME simulation with $n=20$ and $\tau_{\text {grid }}=4$, between 1000 and 2000 days.

produce more variability. Sensitivity tests to these two parameters are presented in the next section.

The imposed cooling profile is plotted in Fig. 3 (left), and the horizontal rms time series of the zonal wind in the lower and upper troposphere (at roughly 800 and $200 \mathrm{hPa}$ ), averaged over the equatorial belt $10^{\circ} \mathrm{S}-10^{\circ} \mathrm{N}$, are plotted in Fig. 3 (right).

In Fig. 4, we plot the Hovmöller diagrams of the meridionally averaged zonal wind in the (left) lower and (middle) upper troposphere and (right) the deep convection heating rate. According to the setting in the multicloud model, the last term can be used as a surrogate for condensation heating and precipitation. In all three panels, planetary-scale/intraseasonal eastwardmoving streaks are clear. The dashed lines indicate the typical $5 \mathrm{~m} \mathrm{~s}^{-1}$ propagation speed of the MJO. Along the dashed lines, westerly wind anomalies at low level, in the left panel, are associated with streaks of easterly winds aloft of about the same magnitude (middle panel) in phase with streaks of enhanced convection as shown in the right panel as seen in nature (Kiladis et al. 2005). We call each such wave pattern an MJO-like event. Along the black dashed line, two long-lived ones can be seen moving in a sequence, close to each other. A train of three short MJO events enter the domain around day 1750 and die around day 1830. Series of successive MJO events are consistent with observations (Yanai et al. 2000; Matthews 2008; Yoneyama et al. 2013) and consistently captured by the stochastic skeleton model (Thual et al. 2014).

A new MJO tracking method has been proposed recently by Zhang et al. (2013b) and Ling et al. (2014), 

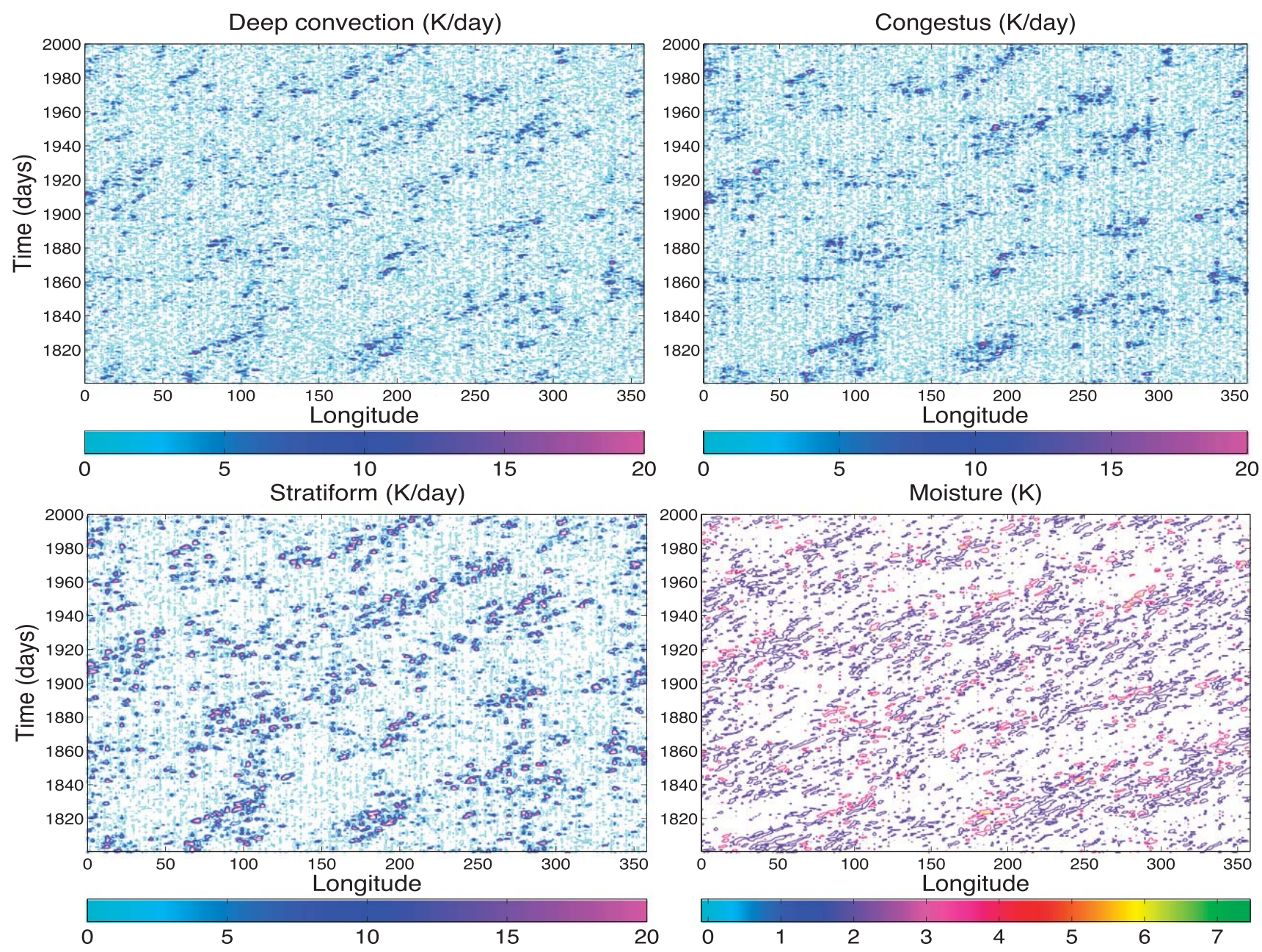

FIG. 6. As in Fig. 4, but for a short period of time between days 1800 and 2000. (top) (left) Deep convection $H_{d}$ and (right) congestus heating $H_{c}$; (bottom) (left) stratiform heating $H_{s}$ and (right) moisture $q$. Entering the domain between 1860 and 1880 days, a train of two MJO-like events move together at roughly $5 \mathrm{~m} \mathrm{~s}^{-1}$.

where the propagation speed is defined as the slope of the track, along which the integrated average of positive precipitation anomalies has the maximum value. We apply this method to the upper-troposphere $(211 \mathrm{hPa})$ zonal wind field, which has more coherent structures, to find the eastward propagation speed of the $31 \mathrm{MJO}$ events occurring between 1000 and 2000 days. The histogram and corresponding Gamma fit curve are shown in Fig. 5. It shows a similar intermittent behavior as in the observation study of Zhang et al. (2013b).

To have a better view of the wave structure, Fig. 6 displays the Hovmöller diagrams of (top left) deep convection, (top right) congestus heating, (bottom left) stratiform heating, and (bottom right) vertically averaged moisture anomalies, between 1800 and 2000 days. The same data are used to compute the power spectra of the meridionally averaged top- and bottom-tropospheric zonal wind, deep convection, and congestus heating that are plotted in Fig. 7. A dominant peak of a planetary-scale-intraseasonal eastward-moving signal is evident on all plots. With respect to time, all the variables show power peaks around the 40-60-day period and wavenumbers 1-5. Notice the horizontally elongated signal suggesting the realistic dispersion relation $d \omega / d k=0$, which is a known characteristic of the MJO (Wheeler and Kiladis 1999; Zhang 2005; Majda and Stechmann 2009).

To get a better demonstration of the dynamical mechanisms of this MJO-like wave, we focus on the single wavenumber-1 MJO-like event between 1600 and 1700 days at first. The Hovmöller diagrams of the corresponding short period are shown in Fig. 8. Inside the MJO-like wave of the heating fields, intermittent large precipitation events are obvious. It is reminiscent of progressive deepening of the convection on multiple scales (Mapes et al. 2006). The physical features of the MJO-like signal in the individual events are confirmed by the horizontal and vertical structure plots (Figs. 9-10) 

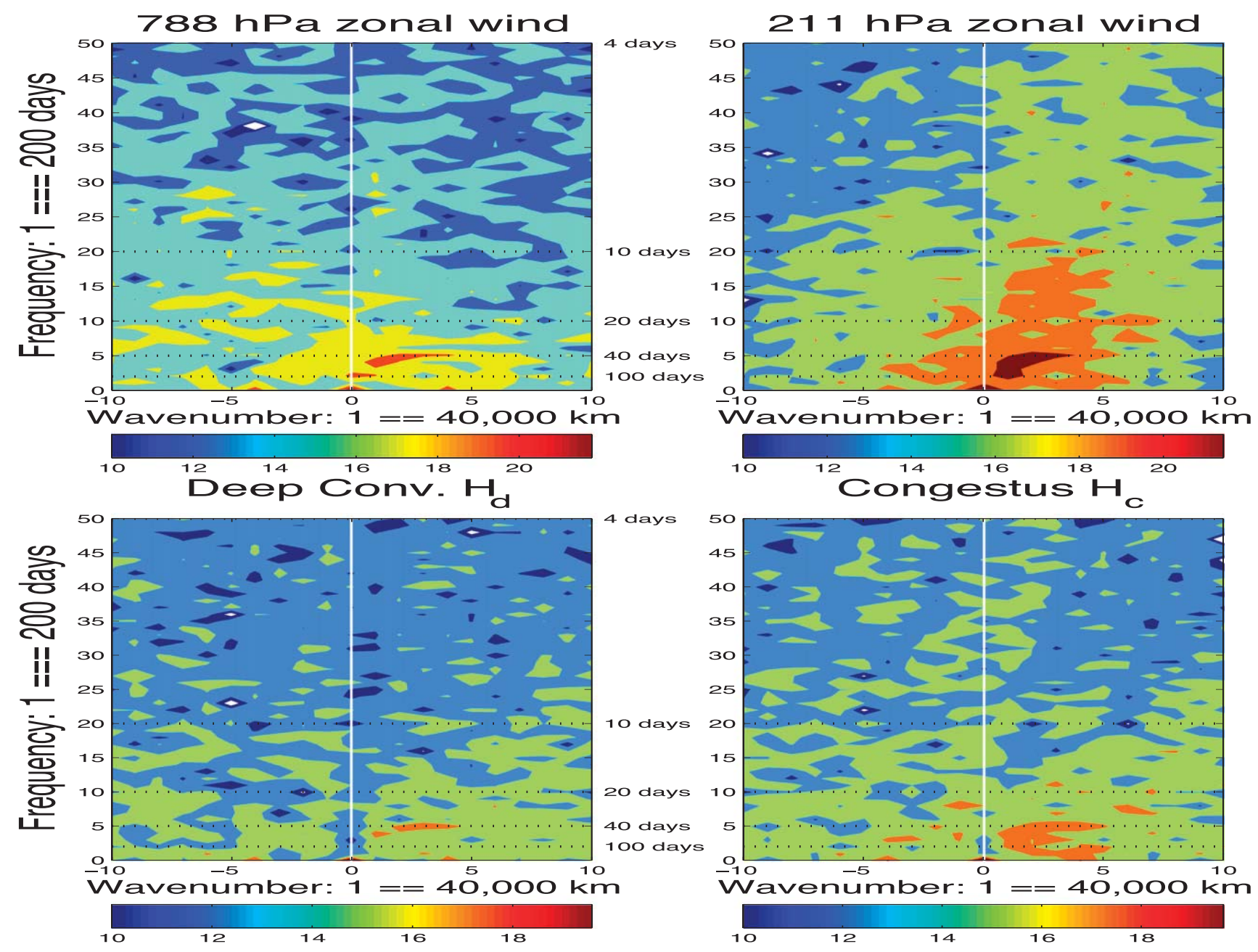

FIG. 7. Logarithm of the spectral power of (top) (left) 788-hPa zonal wind and (right) 211-hPa zonal wind; and (bottom) (left) deep convection $H_{d}$, and (bright) congestus heating $H_{c}$ corresponding to the Hovmöller data in Fig. 6, in the zonal wavenumber-frequency domain, showing the evidence of a planetary-scale-intraseasonal spectral peak on the right side of each panel. The vertical white line marks the boundary between eastward- and westward-moving signals. The corresponding periods are labeled between the panels and are highlighted by the horizontal dashed lines.

of the solution averaged along the frame moving with the wave over the event's life time as indicated by the dashed line in Fig. 4 starting at 1600 days and based on a $5 \mathrm{~m} \mathrm{~s}^{-1}$ phase speed (see KSMT11).

Figure 9 displays the horizontal structure of (left, from top to bottom) MJO-like filtered zonal wind at 250 and $827 \mathrm{hPa}$, deep convection, stratiform and congestus heating, and (right, from top to bottom) relative vorticity with horizontal wind vectors overlaid at 250 and $827 \mathrm{hPa}$, temperature anomalies at $827 \mathrm{hPa}$, moisture, and boundary layer $\theta_{e}$. A single wavenumber-1 MJOlike event is evident, and in the zonal wind field, it is characterized by easterlies leading westerlies at low level and reversed flow aloft. The quadrupole vortex structure is confirmed by both the vorticity contours and the overlaid horizontal wind vectors. A pair of anticyclones (cyclones) is located on both sides of the equator, followed by a pair of cyclones (anticyclones) at low level (aloft). This quadrupole vortex is believed to funnel moisture toward the convection center in the lower troposphere to maintain the wave propagation. The temperature plot suggests that a warm anomaly leads the westerly wind burst (WWB) at the surface. In short, the dynamical features shown in these panels are consistent with the MJO observations in nature (Kiladis et al. 2005; Zhang 2005; Haertel et al. 2008; Kiladis et al. 2009).

Corresponding to the active and suppressed phases of the MJO in the high-OLR observation data, the deep convection is leading and slightly in phase with the surface westerly winds while there is very little deep convective heating in the region of surface easterlies (middle left). Also in agreement with observations, the stratiform heating lags behind the deep convection and the congestus heating anomalies dominate on the north-south 

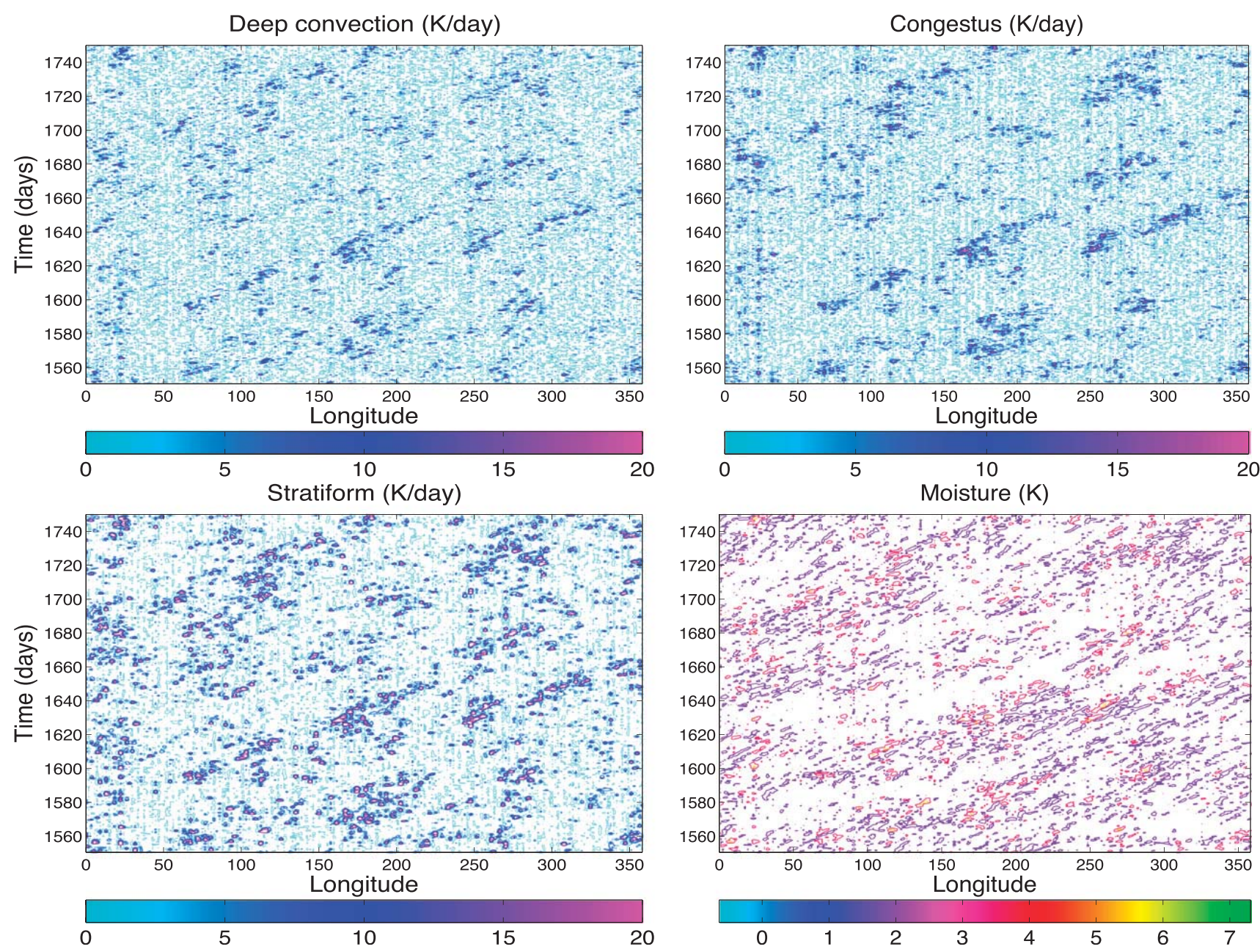

FIG. 8. As in Fig. 4, but for a short period of time between days 1550 and 1750. (top) (left) Deep convection $H_{d}$ and (right) congestus heating $H_{c}$; (bottom) (left) stratiform heating $H_{s}$ and (right) moisture $q$. One strong MJO-like wave starts at about $70^{\circ}$ longitude, between 1580 and 1600 days, moving eastward at roughly $5 \mathrm{~m} \mathrm{~s}^{-1}$, and dies about 60 days later.

flanks of and lead the deep convection center (bottom left). The observed self-similar front-to-rear tilted structure, which characterizes tropical convective systems of all scales (Mapes et al. 2006; Majda 2007; Kiladis et al. 2009), presumably owing to the succession of the heating profiles associated with the three cloud typescongestus, deep, and stratiform-is evident in Fig. 9. Actually, in the MJO events, it is not simply a transition along this sequence but rather a shift in the relative frequency of occurrence of these cloud types and each state lasts 1-2 weeks (Mapes et al. 2006; Del Genio et al. 2012). The constraint of this one-way route from congestus to deep and then to stratiform is used as a design principle for the Markov lattice model in the stochastic multicloud model.

The moisture anomaly structure is a little bit noisy, and there is no significant drying associated with deep convective activity, which may be partially due to the accompanying strong congestus heating on the flanks that supplies off-equatorial moisture through the MJO gyres. The boundary layer $\theta_{e}$ peaks up during the preconditioning phase and exhibits a significant negative anomaly following the deep convection peak associated with CAPE consumption. Note that the linear analysis of the multicloud model on an equatorial beta plane (Khouider and Majda 2008; Han and Khouider 2010) can generate non-equatorial trapped moisture and congestus modes, which may explain the peaking of both moisture and congestus heating off the equator, consistent with the observations (Takayabu et al. 2010).

The vertical structure of the zonal wind (top left), vertical velocity (top right), temperature (bottom left), and total heating (bottom right) are shown in Fig. 10. Below roughly $200 \mathrm{hPa}$, the zonal wind field of this MJOlike wave demonstrates the observed second baroclinic structure (Kiladis et al. 2005). Unlike the DMCMHOMME simulation in KSMT11, the vertical structure of the zonal wind also presents a significant tilt as in 

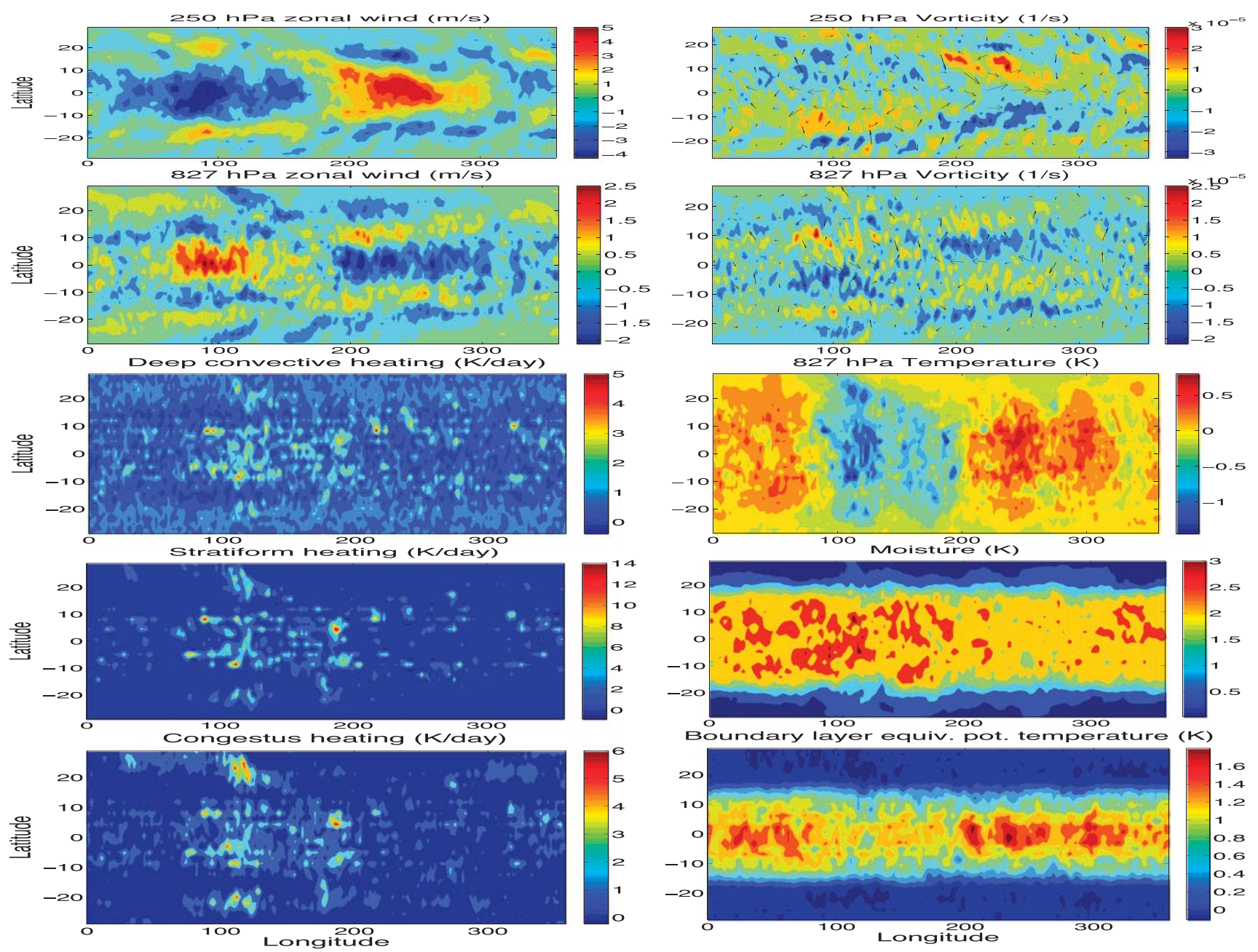

FIG. 9. Horizontal structure of the (left) (top to bottom) MJO-filtered zonal wind at 250 and $827 \mathrm{hPa}$, and deep convection, stratiform, and congestus heating $\left(H_{d}, H_{s}, H_{c}\right.$ ); and (right) (top to bottom) vorticity at 250 and $827 \mathrm{hPa}$ with horizontal velocity vectors overlaid (maximum arrow is 4.3538 and $2.6442 \mathrm{~m} \mathrm{~s}^{-1}$, respectively), temperature anomalies at $827 \mathrm{hPa}$, moisture, and boundary layer $\theta_{e}$, for the single MJO-like event following the dashed line in Fig. 4 starting at 1600 days.

the observation. Because of the parameter setting, the congestus and stratiform heating are strong in this case (as shown in Figs. 6 and 8-10), which can explain this significant tilt. The warm temperatures are leading and slightly in phase with deep convection, followed by the cold anomalies, which dominate the suppressed region. As expected, the vertical velocity is in phase with the heating: upward motions in the heating regions and downward motion in the cooling regions.

Figure 11 shows some structure of the train of two wavenumber-2 $\mathrm{MJO}$ events along the black dashed line in Fig. 4. Note that a strong MJO is leading a weak one, as in the simulation of KSMT11, consistent with the Tropical Ocean Global Atmosphere Coupled OceanAtmosphere Response Experiment (TOGA COARE) MJOs (Yanai et al. 2000). Other individual features are similar to the single wavenumber-1 MJO event, consistent with the claimed self-similarity of convectively coupled tropical waves (Mapes et al. 2006; Majda 2007; Kiladis et al. 2009).

\section{Sensitivity tests and statistical analysis \\ a. Sensitivity tests to $n$ and $\tau_{\text {grid }}$}

In the stochastic lattice model, each GCM grid box is occupied by $n \times n$ sites and the transition time scales are set to vary with the number of lattice sites or equivalently with the GCM grid size through the parameter $\tau_{\text {grid }}$ (FMK13). Here, we discuss the sensitivity of the stochastic simulations to changes in $\tau_{\text {grid }}$ and $n$.

The stochastic multicloud model uses the congestus, deep, and stratiform cloud-type triplet as the "building block" to describe the dynamics of convective clusters (Mapes et al. 2006). With the GCM grid size fixed, smaller $n$ means bigger spatial size of the building block, and bigger $\tau_{\text {grid }}$ means longer life cycle (FMK12). 

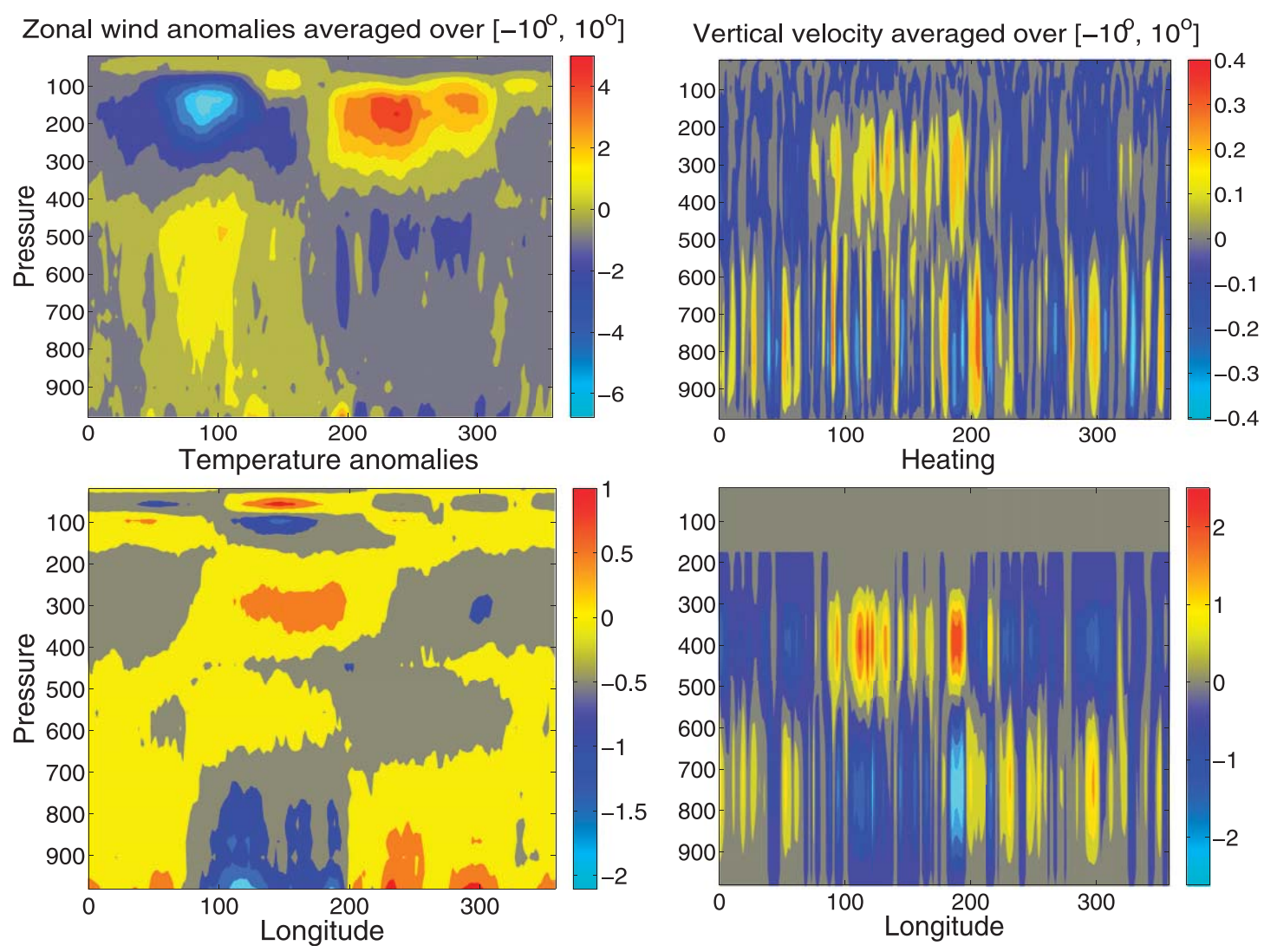

FIG. 10. Vertical structure of the MJO-filtered (left) (top) zonal wind $\left(\mathrm{m} \mathrm{s}^{-1}\right)$ and (bottom) temperature anomalies (K); and (right) (top) vertical velocity $\left(\mathrm{m} \mathrm{s}^{-1}\right)$, and (bottom) total heating $\left(\mathrm{K} \mathrm{day}^{-1}\right)$, for the single MJO-like event as in Fig. 9.

Accordingly, by definition of the transition rates, both smaller $n$ and bigger $\tau_{\text {grid }}$ correspond to longer transition time scales.

We consider the following four cases. Three corresponding to a fixed number of lattice sites, $n=20$, and time scale dilation parameter $\tau_{\text {grid }}=1,4$, and 8 , respectively, while the fourth uses $\tau_{\text {grid }}=4$ and $n=10$. The $x-t$ contours of the meridionally averaged zonal wind in the upper troposphere of these four test cases are shown in Fig. 12. Note that the case $n=20, \tau_{\text {grid }}=4$ corresponds to our standard case reported in the previous section and has clear MJO events. When $\tau_{\text {grid }}=1$, $n=20$, fast Kelvin waves dominate the system and no clear MJO events are produced (Fig. 12, top right). Some MJO events are generated in the case with $\tau_{\text {grid }}=$ $8, n=20$ (Fig. 12, bottom left), but the structure is very noisy, compared with the case with $\tau_{\text {grid }}=4$. Also, when we set $\tau_{\text {grid }}=4$ and decrease the number of cloud sites to $n=10$ (Fig. 12, bottom right), the system generates some noisy MJO waves, similar to the case with $(n=20$, $\left.\tau_{\text {grid }}=8\right)$.

Overall, the transition time scales corresponding to $\tau_{\text {grid }}=4$ constitutes a balanced regime with just enough variability consistent with the results in FMK12. A small number of cloud sites can be used to increase the variability of the system but a too-small $n$ will also make the wave structure very noisy. On the other hand, consistent with earlier studies (e.g., Majda and Khouider 2002; FMK12; FMK13), increasing the number of convective elements in the stochastic model leads to the mean field limit; thereby yielding deterministic behavior. Here, with $n=120$ and $n=160$ (results not shown), the system generates only Kelvin waves as in the case of the deterministic parameterization.

\section{b. Statistical analysis}

One difficulty for GCMs is to reproduce the statistics of tropical rainfall and precipitable water (Neelin et al. 2008; Holloway and Neelin 2009). While it has proven difficult so far for deterministic GCMs to exhibit both the observed fat tails of rainfall and correct autocorrelation times of both rainfall and water vapor, it is argued in Neelin et al. (2008) that these are test beds for GCMs with stochastic parameterization. It is shown in FMK13 that despite the highly simplified dynamical core of the toy GCM used there, the SMCM was capable in reproducing qualitatively some features of these statistics. Here, we extend this analysis to the HOMME-SMCM simulations. 

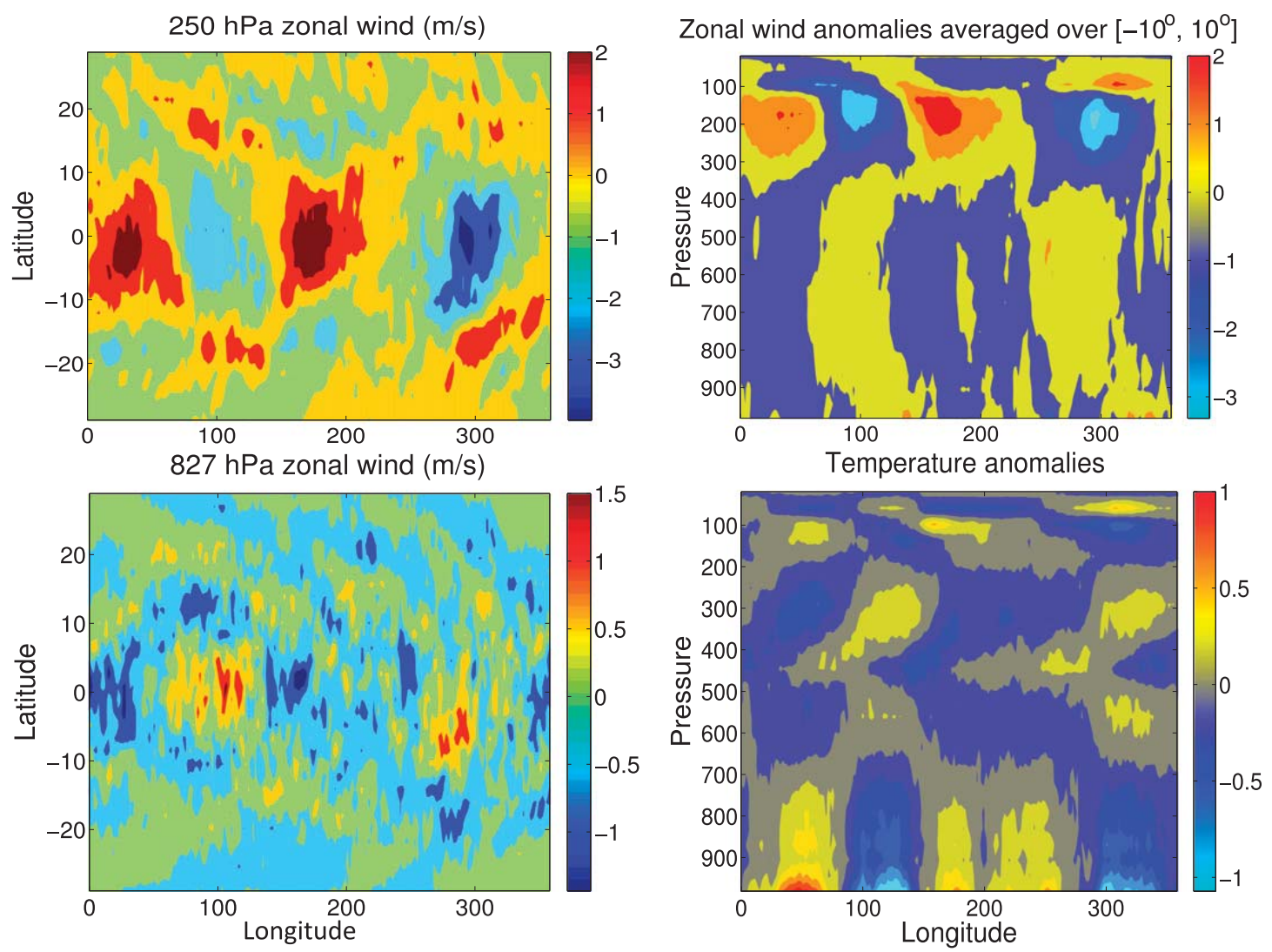

FIG. 11. Horizontal structure of the MJO-filtered zonal wind at (left) (top) $250 \mathrm{hPa}$ and (bottom) $827 \mathrm{hPa}$. Vertical structure of the MJO-filtered (right) (top) zonal wind $\left(\mathrm{m} \mathrm{s}^{-1}\right.$ ) and (bottom) temperature anomalies (K) for the train of two wavenumber-2 MJO-like events along the black dashed line in Fig. 4.

To collect such statistics we adopt the algorithm used in FMK13. The simulations are sampled at fixed grid points, which are viewed as "observation sites" (FMK13). The associated time series, from individual sites along the equator, are then connected to produce one long time series. To ensure the lack of significant correlations in the resulting time series, the spacing between two adjacent sites is set to $2000 \mathrm{~km}$ (FMK13). To mimic the typical setting in radiosonde and satellite observations (Holloway and Neelin 2009), the sampling rate of $1 \mathrm{~h}$ is chosen. Only the data from 500 to 2000 days of the simulations is used; the first-transient 500 days were discarded.

Following FMK13, the first statistical measure considered here is the distribution of the precipitation events. Figure 13 displays the results of the four simulations with different lattice numbers $(n)$ and transition time scales $\left(\tau_{\text {grid }}\right)$ used in the sensitivity test above. Here, the frequency of occurrence is plotted as a function of the total precipitation $(\mathrm{mm})$ in each event. To convert heating rates to $\mathrm{mm}$, we use the fact that $4 \mathrm{~mm}$ of rain corresponds roughly to heating one $m^{2}$-tropospheric column of air by $1 \mathrm{~K}$, based on the energy conservation principle, under normal atmospheric conditions (FMK13). Interestingly, for the cases where MJOs are reproduced (top left, bottom left, and bottom right of Fig. 13), two different power laws are obvious on each plot. For precipitation events greater than $10 \mathrm{~mm}$, the occurrence frequency falls approximately by 2 factors of 10 when the total precipitation increases by about 1 factor of 10 ; a relatively flat power laws can be observed for the moderate precipitation events $(1-10 \mathrm{~mm})$. The twopower-law structure is consistent with the observational studies of Neelin et al. (2008) and Peters et al. (2010). The fat tails in these three cases reflect the highly intermittent strong precipitation events consistent with the observed high variability, while in the simulations without clear MJO $\left(n=20, \tau_{\text {grid }}=1\right)$ (top right of Fig. 13), only one steep power law is observed.

The second statistical measure considered here is the autocorrelation function for the vertically averaged moisture and total precipitation. Here, results of the simulations with $\left(n=20, \tau_{\text {grid }}=4\right)$ and $\left(n=20, \tau_{\text {grid }}=1\right)$ are shown in Fig. 14 (top left and top right). Qualitatively consistent with the observational studies in Holloway and Neelin $(2009,2010)$, the autocorrelation of the precipitation is much shorter than the moisture. Also as expected, in the simulation where clear MJOs are produced $\left(n=20, \tau_{\text {grid }}=4\right)$, the variables have longer 

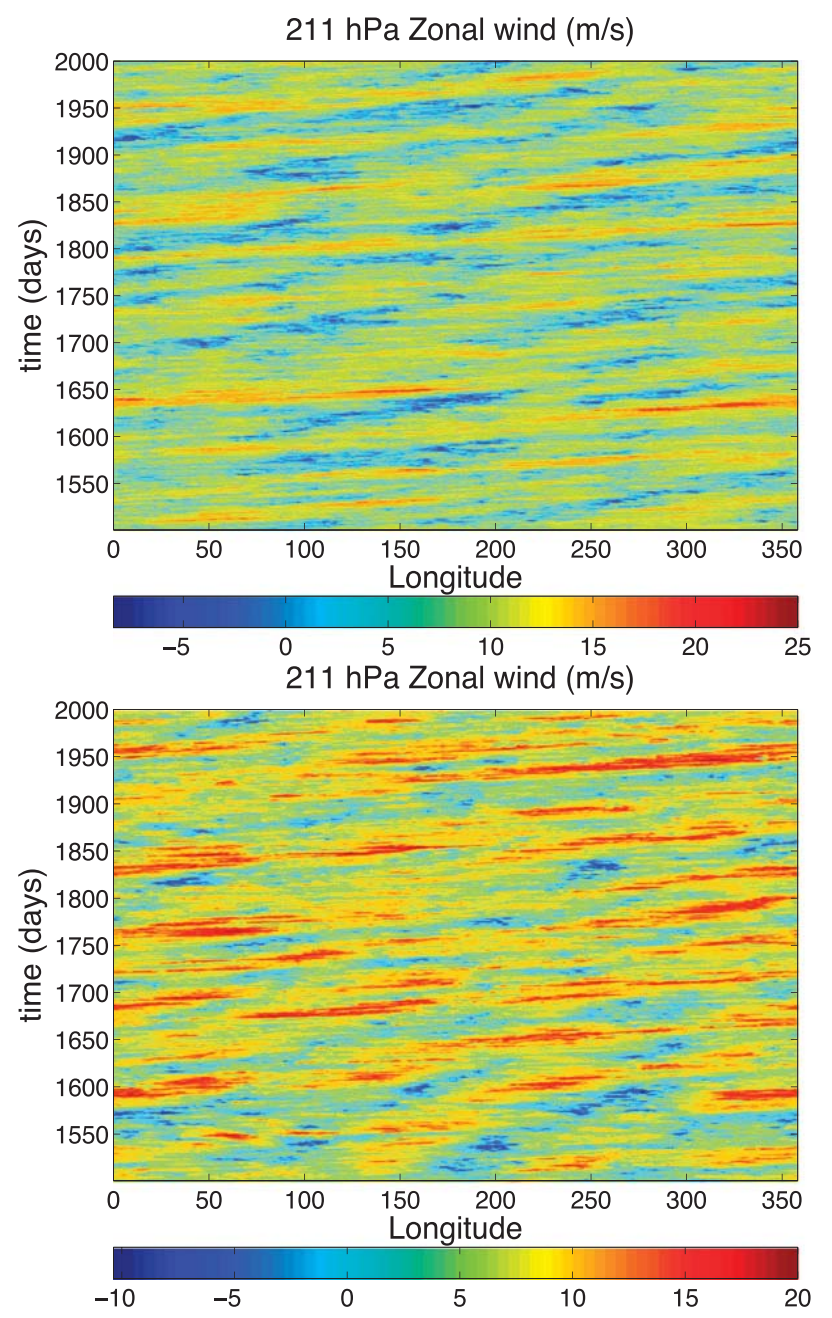
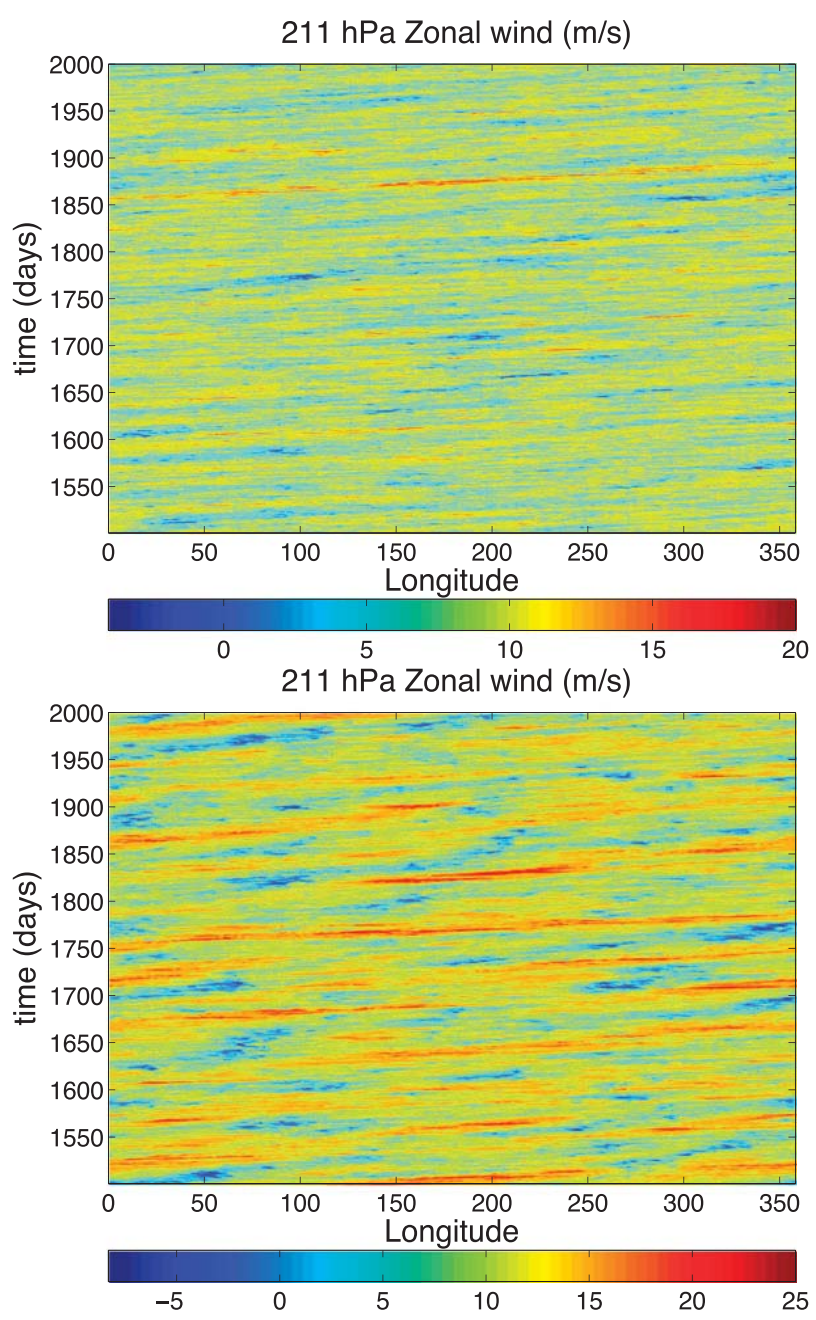

FIG. 12. Hovmöller diagrams of the top-of-troposphere zonal wind for the SMCM-HOMME simulations with parameters as in Table 3 but different number of lattice sites $(n)$ and transition time scale parameter $\left(\tau_{\text {grid }}\right)$. (top) (left) $n=20, \tau_{\text {grid }}=4$ and (right) $n=20, \tau_{\text {grid }}=1$; (bottom) (left) $n=20, \tau_{\text {grid }}=8$ and (right) $n=10, \tau_{\text {grid }}=4$.

autocorrelation, since bigger $\tau_{\text {grid }}$ means longer transition time for the cloud system and MJO is a phenomenon with large spatial and temporal scales. In the observational studies of Holloway and Neelin (2010), the $e$-folding time of the precipitation is on the order of $1 \mathrm{~h}$ or less while the for column water vapor, it is around 2 days or more. In our simulations, the $e$-folding time of precipitation varies between 2 and $5 \mathrm{~h}$ and that of water vapor is roughly 1 day for the top two panels. The bottom two plots of Fig. 14 use the data averaged in a horizontal $5 \times 5$ grid box $\left(800 \times 800 \mathrm{~km}^{2}\right.$ domain $)$ centered at each sampling site to mimic the large averaging areas used in the observations. An interesting feature is the spatial self-similarity of the precipitation. With the horizontal domain averaging (bottom two plots of Fig. 14), the autocorrelation curve of the precipitation is almost the same while the $e$-folding time of the moisture has almost doubled to roughly match the observations.
Overall, the results shown here are consistent with those of Stechmann and Neelin (2011) where an "external" potential is used to force a simple stochastic switching system in order to reproduce the observed statistical properties of large precipitation events. The SMCM can generate such forcing naturally through its own internal dynamics (also see FMK13). Note that we focus on MJO events in this paper where the system gets into the statistical equilibrium relatively fast (see Fig. 3, left), while in the observational studies papers the data of more general tropical conditions were gathered providing a wider variability spectrum.

\section{Concluding summary and discussion}

Here the High-Order Methods Modeling Environment (HOMME) NCAR GCM is coupled to the stochastic multicloud convective parameterization (KBM10; FMK12). This SMCM-HOMME model is tested in an aquaplanet 

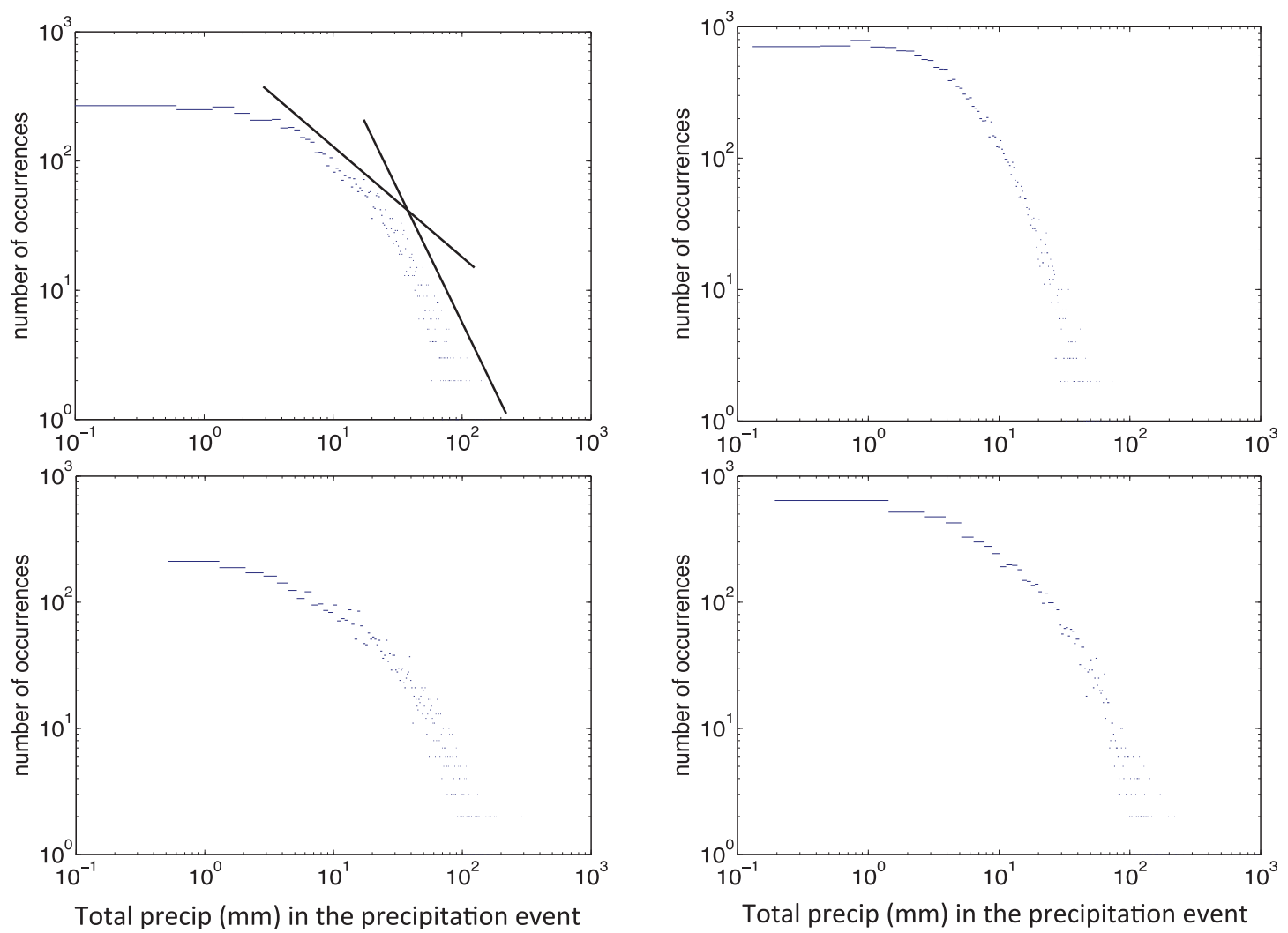

FIG. 13. Distribution of the precipitation events for the SMCM-HOMME simulations with different $n$ and $\tau_{\text {grid }}$ as in Fig. 12. (top) (left) $n=20, \tau_{\text {grid }}=4$ and (right) $n=20, \tau_{\text {grid }}=1$; (bottom) (left) $n=20, \tau_{\text {grid }}=8$ and (right) $n=10$, $\tau_{\text {grid }}=4$.

setting with the parameter regime, which has been used for the deterministic multicloud-HOMME model in KSMT11. As in the case of the simple toy GCM used in FMK12, the stochastic parameterization drastically improves the variability and character of simulated convectively coupled wave patterns. In place of very regular convectively coupled waves, the SMCM-HOMME reproduces highly intermittent chaotic MJO-like events (Figs. 4-11).

The typical $5 \mathrm{~m} \mathrm{~s}^{-1}$ propagation speed of the MJO is captured and the peaks in the spectral power plots of zonal wind and heating fields are consistent with observations. A manifestation of intermittent stochastic behavior of MJO variability can be seen in the various types of MJO events that are reproduced. Sometimes there is a single wavenumber-1 MJO event, sometimes there is a train of two wavenumber-2 MJO events, and sometimes a train of three MJO events are seen, as it often occurs in nature (Matthews 2008) and also consistent with the results obtained by a simple theoretical model: namely, the stochastic MJO-skeleton model (Thual et al. 2014). The propagation speed of the MJOlike events varies roughly between 3 to $9 \mathrm{~m} \mathrm{~s}^{-1}$ and has a distribution very similar to observations (Zhang et al. 2013b). Furthermore, it is shown that the SMCMHOMME can capture qualitatively two observed statistical features of tropical precipitation (Neelin et al. 2008; Peters et al. 2010). First, the precipitation event size distribution has two power laws. Second, moisture and precipitation have long (days) and short (hours) autocorrelation times, respectively.

Consistent with the arguments in FMK13, the stochastic parameterization improves the deterministic parameterization results mainly because the deterministic model is not capable to produce enough chaotic behavior; the SMCM is a good emulator of organized convection variability. As demonstrated in Peters et al. (2013), the SMCM replicates well the highly nonlinear character of tropical precipitation variability, which is characterized by an inversely proportional noise to mean dependence. Such behavior cannot be captured by a linearly tuned noise model as in Buizza et al. (1999) or Lin and Neelin (2003) or by a simple Poisson process as in Plant and Craig (2008). Here, the main difference between the stochastic and deterministic parameterization is the variability induced by the cloud area fractions through the Markov chain lattice model, which is designed to capture the systematic transitions between the various cloud 

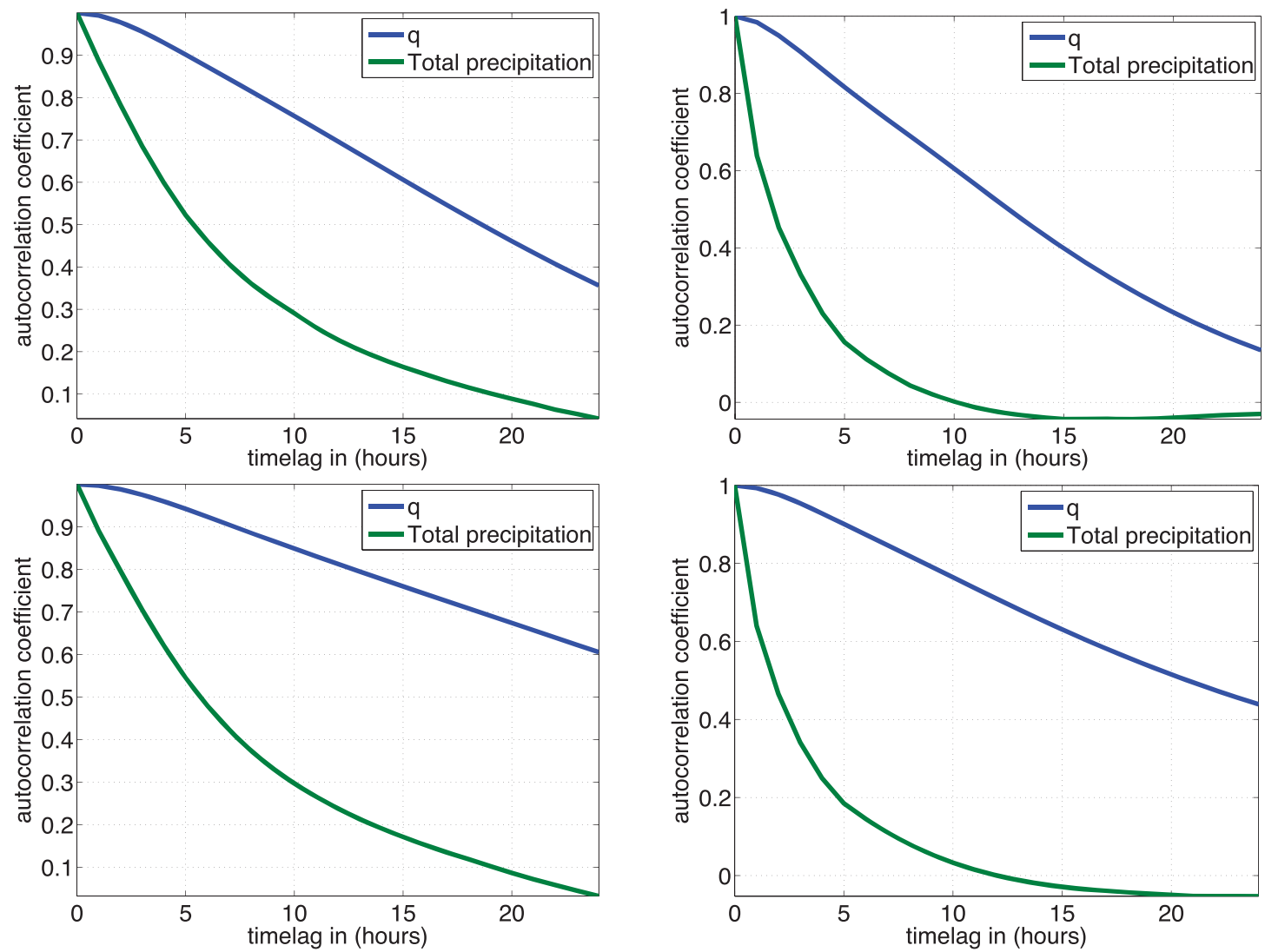

FIG. 14. Autocorrelation of the moisture and total precipitation for the SMCM-HOMME simulations. The bottom two panels used data that were averaged in a horizontal $5 \times 5$ grid box $\left(800 \times 800 \mathrm{~km}^{2}\right.$ domain $)$ centered at each sampling site. (top) (left) $n=20, \tau_{\text {grid }}=4$, and (right) $n=20, \tau_{\text {grid }}=1$; (bottom) (left) $n=20, \tau_{\text {grid }}=4$, with averaging and (right) $n=20, \tau_{\text {grid }}=1$, with averaging.

types, essentially from congestus to deep and from deep to stratiform. Sensitivity tests and statistical analysis confirmed that the SMCM-HOMME simulations are sensitive to the number of convective elements and transition time scales. To reproduce clear MJO-like structures, we need to tune these two parameters carefully.

Peters et al. (2013) visually constrained the equilibrium distribution of the SMCM cloud area fractions to radar spectrometry data to find "best-fit" transition time scales that better represent the statistics of the rainfall time series for two particular cases of tropical precipitation over and around two islands (Darwin and Kwajalein). While the SMCM successfully passed this comparative study, the same transition time scales performed poorly in the SMCM-HOMME in terms of producing clear MJO simulation. This is not very surprising since in addition to the reasons already mentioned (in the introduction) the dynamics of convection within the MJO and over the particular sites may be very different. Whereas the current simulations are on an aquaplanet, the parameters in Peters et al. (2013) considered Darwin, where considerable land-sea interactions are exhibited. Thus proper "tuning" of the SMCM parameters within different GCM settings is crucial. Maybe a one-forall set of parameters, even within the same GCM, is not the solution.

Finally, it is important to emphasize that SMCMHOMME produces MJOs with numerous realistic features from nature [including the notorious front-to-rear vertical tilt and quadruple vortex structure (Kiladis et al. 2005)] based only on the correct phasing of three cloud types of random convective organization on mesoscales and synoptic scales. In particular, there is no wind induced surface heat exchange, no cloud radiative feedback, nor air-sea coupling in the simulations here, in contrast to the various theories that postulate their important role in driving the MJO (Lau and Waliser 2012).

Acknowledgments. The Center for Prototype Climate Modeling (CPCM) is fully funded by the Abu Dhabi Government through a New York University Abu Dhabi (NYUAD) Research Institute grant. This research was initiated during an extended visit of B.K. and A.J.M. to the CPCM at NYUAD during spring 2013. 
The computations were carried out on the High Performance Computing resources at NYUAD. The research of B.K. is partially funded by the Natural and Engineering Research Council of Canada. The research of A.J.M. is partially supported by the Office of Naval Research Grant ONR MURI N00014-12-1-0912.

\section{REFERENCES}

Ajayamohan, R. S., B. Khouider, and A. J. Majda, 2013: Realistic initiation and dynamics of the Madden-Julian oscillation in a coarse resolution aquaplanet GCM. Geophys. Res. Lett., 40, 6252-6257, doi:10.1002/2013GL058187.

Buizza, R., M. Milleer, and T. N. Palmer, 1999: Stochastic representation of model uncertainties in the ECMWF ensemble prediction system. Quart. J. Roy. Meteor. Soc., 125, 2887-2908, doi:10.1002/qj.49712556006.

Crueger, T., B. Stevens, and R. Brokopf, 2013: The Madden-Julian oscillation in ECHAM6 and the introduction of an objective MJO metric. J. Climate, 26, 3241-3256, doi:10.1175/ JCLI-D-12-00413.1.

De La Chevrotiere, M., B. Khouider, and A. Majda, 2014: Calibration of the stochastic multicloud model using Bayesian inference. SIAM J. Sci. Comput., 36, B538-B560, doi:10.1137/ $13094267 \mathrm{X}$.

Del Genio, A. D., Y. Chen, D. Kim, and M.-S. Yao, 2012: The MJO transition from shallow to deep convection in CloudSat/ CALIPSO data and GISS GCM simulations. J. Climate, 25, 3755-3770, doi:10.1175/JCLI-D-11-00384.1.

Frenkel, Y., A. J. Majda, and B. Khouider, 2012: Using the stochastic multicloud model to improve tropical convective parameterization: A paradigm example. J. Atmos. Sci., 69,10801105, doi:10.1175/JAS-D-11-0148.1.

,-- , and -2013 : Stochastic and deterministic multicloud parameterizations for tropical convection. Climate Dyn., 41, 1527-1551, doi:10.1007/s00382-013-1678-z.

Gillespie, D. T., 1975: An exact method for numerically simulating the stochastic coalescence process in a cloud. J. Atmos. Sci., 32, 19771989, doi:10.1175/1520-0469(1975)032<1977:AEMFNS > 2.0.CO;2.

- 1977: Exact stochastic simulation of coupled chemical reactions. J. Phys. Chem., 81, 2340-2361, doi:10.1021/j100540a008.

Grabowski, W. W., 2002: Large-scale organization of moist convection in idealized aquaplanet simulations. Int. J. Numer. Methods Fluids, 39, 843-853, doi:10.1002/fld.332.

— , and M. W. Moncrieff, 2004: Moisture-convection feedback in the tropics. Quart. J. Roy. Meteor. Soc., 130, 3081-3104, doi:10.1256/qj.03.135.

Haertel, P. T., G. N. Kiladis, A. Denno, and T. M. Rickenbach, 2008: Vertical-mode decomposition of 2-day waves and the Madden-Julian oscillation. J. Atmos. Sci., 65, 813-833, doi:10.1175/ 2007JAS2314.1.

Han, Y., and B. Khouider, 2010: Convectively coupled waves in a sheared environment. J. Atmos. Sci., 67, 2913-2942, doi:10.1175/2010JAS3335.1.

Holloway, C. E., and J. D. Neelin, 2009: Moisture vertical structure, column water vapor, and tropical deep convection. J. Atmos. Sci., 66, 1665-1683, doi:10.1175/2008JAS2806.1.

$\longrightarrow$, and - 2010: Temporal relations of column water vapor and tropical precipitation. J. Atmos. Sci., 67, 1091-1105, doi:10.1175/2009JAS3284.1.

Johnson, R. H., T. M. Rickenbach, S. A. Rutledge, P. E. Ciesielski, and W. H. Schubert, 1999: Trimodal characteristics of tropical convection. J. Climate, 12, 2397-2418, doi:10.1175/ 1520-0442(1999)012<2397:TCOTC>2.0.CO;2.

Kasahara, A., and K. Puri, 1981: Spectral representation of threedimensional global data by expansion in normal mode functions. Mon. Wea. Rev., 109,37-51, doi:10.1175/1520-0493(1981)109<0037: SROTDG $>2.0 . \mathrm{CO} ; 2$.

Katsoulakis, M. A., A. J. Majda, and D. G. Vlachos, 2003: Coarsegrained stochastic processes and Monte Carlo simulations in lattice systems. J. Comput. Phys., 186, 250-278, doi:10.1016/ S0021-9991(03)00051-2.

Khouider, B., 2014: A coarse grained stochastic multi-type particle interacting model for tropical convection: Nearest neighbour interactions. Commun. Math. Sci., 12,1379-1407, doi:10.4310/ CMS.2014.v12.n8.a1.

_ , and A. J. Majda, 2006a: Model multi-cloud parameterizations for convectively coupled waves: Detailed nonlinear wave evolution. Dyn. Atmos. Oceans, 42, 59-80, doi:10.1016/ j.dynatmoce.2005.12.001.

- and - 2006b: A simple multicloud parameterization for convectively coupled tropical waves. Part I: Linear analysis. J. Atmos. Sci., 63, 1308-1323, doi:10.1175/JAS3677.1.

— , and — 2008: Equatorial convectively coupled waves in a simple multicloud model. J. Atmos. Sci., 65, 3376-3397, doi:10.1175/2008JAS2752.1.

,$-\ldots$, and M. A. Katsoulakis, 2003: Coarse-grained stochastic models for tropical convection and climate. Proc. Natl. Acad. Sci. USA, 100, 11 941-11946, doi:10.1073/pnas.1634951100.

, J. Biello, and A. J. Majda, 2010: A stochastic multicloud model for tropical convection. Comm. Math. Sci., 8, 187-216, doi:10.4310/CMS.2010.v8.n1.a10.

— , A. St-Cyr, A. J. Majda, and J. Tribbia, 2011: MJO and convectively coupled waves in a coarse resolution GCM with a simple multicloud parametrization. J. Atmos. Sci., 68, 240264, doi:10.1175/2010JAS3443.1.

_ A. J. Majda, and S. N. Stechmann, 2013: Climate science in the tropics: Waves, vortices and PDEs. Nonlinearity, 26, R1, doi:10.1088/0951-7715/26/1/R1.

Kikuchi, K., and Y. Takayabu, 2004: The development of organized convection associated with the MJO during TOGA COARE IOP: Trimodal characteristics. Geophys. Res. Lett., 31, L10101, doi:10.1029/2004GL019601.

Kiladis, G. N., K. Straub, and P. Haertel, 2005: Zonal and vertical structure of the Madden-Julian oscillation. J. Atmos. Sci., 62, 2790-2809, doi:10.1175/JAS3520.1.

—, M. C. Wheeler, P. T. Haertel, K. H. Straub, and P. E. Roundy, 2009: Convectively coupled equatorial waves. Rev. Geophys., 47, RG2003, doi:10.1029/2008RG000266.

Kim, D., and Coauthors, 2009: Application of MJO simulation diagnostics to climate models. J. Climate, 22, 6413-6436, doi:10.1175/2009JCLI3063.1.

Krishnamurti, T. N., H. S. Bedi, V. M. Hardiker, and L. Ramaswamy, 2006: An Introduction to Global Spectral Modeling. Springer, $272 \mathrm{pp}$.

Lau, W. K.-M., and D. E. Waliser, 2012: Intraseasonal Variability in the Atmosphere-Ocean Climate System. 2nd ed. Springer Praxis Books, $614 \mathrm{pp}$.

Lin, J.-L., B. Mapes, M. Zhang, and M. Newman, 2004: Stratiform precipitation, vertical heating profiles, and the Madden-Julian oscillation. J. Atmos. Sci., 61, 296-309, doi:10.1175/ 1520-0469(2004)061<0296:SPVHPA > 2.0.CO;2.

-, and Coauthors, 2006: Tropical intraseasonal variability in 14 IPCC AR4 climate models. Part I: Convective signals. J. Climate, 19, 2665-2690, doi:10.1175/JCLI3735.1. 
Lin, J. W.-B., and J. D. Neelin, 2003: Toward stochastic deep convective parameterization in general circulation models. Geophys. Res. Lett., 30, 1162, doi:10.1029/2002GL016203.

Lin, X., and R. H. Johnson, 1996: Kinematic and thermodynamic characteristics of the flow over the western Pacific warm pool during TOGA COARE. J. Atmos. Sci., 53, 695-715, doi:10.1175/1520-0469(1996)053<0695:KATCOT>2.0.CO;2.

Ling, J., P. Bauer, P. Bechtold, A. Beljaars, R. Forbes, F. Vitart, M. Ulate, and C. Zhang, 2014: Global versus local MJO forecast skill of the ECMWF model during DYNAMO. Mon. Wea. Rev., 142, 2228-2247, doi:10.1175/MWR-D-13-00292.1.

Liu, P., and Coauthors, 2009: Tropical intraseasonal variability in the MRI-20km60L AGCM. J. Climate, 22, 2006-2022, doi:10.1175/2008JCLI2406.1.

Madden, R., and P. R. Julian, 1972: Description of global-scale circulation cells in the tropics with a 40-50-day period. J. Atmos. Sci., 29, 1109-1123, doi:10.1175/1520-0469(1972)029<1109: DOGSCC $>2.0 . \mathrm{CO} ; 2$.

Majda, A. J., 2007: New multiscale models and self-similarity in tropical convection. J. Atmos. Sci., 64, 1393-1404, doi:10.1175/ JAS3880.1.

— , and M. Shefter, 2001: Models for stratiform instability and convectively coupled waves. J. Atmos. Sci., 58, 1567-1584, doi:10.1175/1520-0469(2001)058<1567:MFSIAC >2.0.CO;2.

— , and B. Khouider, 2002: Stochastic and mesoscopic models for tropical convection. Proc. Natl. Acad. Sci. USA, 99, 1123-1128, doi:10.1073/pnas.032663199.

— , and S. N. Stechmann, 2009: The skeleton of tropical intraseasonal oscillations. Proc. Natl. Acad. Sci. USA, 106, $8417-$ 8422, doi:10.1073/pnas.0903367106.

—- B. Khouider, G. N. Kiladis, K. H. Straub, and M. G. Shefter, 2004: A model for convectively coupled tropical waves: Nonlinearity, rotation, and comparison with observations. J. Atmos. Sci., 61, 2188-2205, doi:10.1175/ 1520-0469(2004)061<2188:AMFCCT>2.0.CO;2.

Mapes, B. E., 2000: Convective inhibition, subgrid-scale triggering energy, and stratiform instability in a toy tropical wave model. J. Atmos. Sci., 57, 1515-1535, doi:10.1175/ 1520-0469(2000)057<1515:CISSTE > 2.0.CO;2.

—, S. Tulich, J. Lin, and P. Zuidema, 2006: The mesoscale convection life cycle: Building block or prototype for large-scale tropical waves? Dyn. Atmos. Oceans, 42, 3-29, doi:10.1016/ j.dynatmoce.2006.03.003.

Matthews, A. J., 2008: Primary and successive events in the MaddenJulian oscillation. Quart. J. Roy. Meteor. Soc., 134, 439-453, doi:10.1002/qj.224.

Moncrieff, M. W., and E. Klinker, 1997: Organized convective systems in the tropical western Pacific as a process in general circulation models: A TOGA COARE case-study. Quart. J. Roy. Meteor. Soc., 123, 805-827, doi:10.1002/qj.49712354002.

Neelin, J. D., O. Peters, J. W.-B. Lin, K. Hales, and C. E. Holloway, 2008: Rethinking convective quasi-equilibrium: Observational constraints for stochastic convective schemes in climate models. Philos. Trans. Roy. Soc. London, A366, 2579-2602, doi:10.1098/rsta.2008.0056.

Peters, K., C. Jakob, L. Davies, B. Khouider, and A. Majda, 2013: Stochastic behavior of tropical convection in observations and a multicloud model. J. Atmos. Sci., 70,3556-3575, doi:10.1175/ JAS-D-13-031.1.

Peters, O., A. Deluca, A. Corral, J. D. Neelin, and C. E. Holloway, 2010: Universality of rain event size distributions. J. Stat. Mech.: Theory Exp., 2010, P11030, doi:10.1088/1742-5468/2010/11/ P11030.

Plant, R. S., and G. C. Craig, 2008: A stochastic parameterization for deep convection based on equilibrium statistics. J. Atmos. Sci., 65, 87-105, doi:10.1175/2007JAS2263.1.

Stechmann, S. N., and J. D. Neelin, 2011: A stochastic model for the transition to strong convection. J. Atmos. Sci., 68, 2955-2970, doi:10.1175/JAS-D-11-028.1.

Stensrud, D. J., 2007: Parametrization Schemes: Keys to Understanding Numerical Weather Prediction Models. Cambridge University Press, 478 pp.

Subramanian, A. C., M. Jochum, A. J. Miller, R. Murtugudde, R. B. Neale, and D. E. Waliser, 2011: The Madden-Julian oscillation in CCSM4. J. Climate, 24, 6261-6282, doi:10.1175/ JCLI-D-11-00031.1.

Takayabu, Y. N., S. Shige, W.-K. Tao, and N. Hirota, 2010: Shallow and deep latent heating modes over tropical oceans observed with TRMM PR spectral latent heating data. J. Climate, 23, 2030-2046, doi:10.1175/2009JCLI3110.1.

Thual, S., A. J. Majda, and S. N. Stechmann, 2014: A stochastic skeleton model for the MJO. J. Atmos. Sci., 71, 697-715, doi:10.1175/JAS-D-13-0186.1.

Wheeler, M., and G. N. Kiladis, 1999: Convectively coupled equatorial waves: Analysis of clouds and temperature in the wavenumber-frequency domain. J. Atmos. Sci., 56, 374-399, doi:10.1175/1520-0469(1999)056<0374:CCEWAO>2.0.CO;2.

Yanai, M., B. Chen, and W. Tung, 2000: The Madden-Julian oscillation observed during the TOGA COARE IOP: Global view. J. Atmos. Sci., 57, 2374-2396, doi:10.1175/ 1520-0469(2000)057<2374:TMJOOD>2.0.CO;2.

Yoneyama, K., C. Zhang, and C. N. Long, 2013: Tracking pulses of the Madden-Julian oscillation. Bull. Amer. Meteor. Soc., 94, 1871-1891, doi:10.1175/BAMS-D-12-00157.1.

Zhang, C., 2005: Madden-Julian Oscillation. Rev. Geophys., 43, RG2003, doi:10.1029/2004RG000158.

—, J. Gottschalck, E. Maloney, M. Moncrieff, F. Vitart, D. Waliser, B. Wang, and M. Wheeler, 2013a: Cracking the MJO nut. Geophys. Res. Lett., 40, 1223-1230, doi:10.1002/ grl.50244.

_ - M. Ulate, and J. Liang, cited 2013b: A MJO tracking method. [Available online at http://abstractsearch.agu.org/meetings/ 2013/FM/sections/A/sessions/A31F/abstracts/A31F-0146. html.] 